\title{
MicroRNA-124 Attenuates PTSD-like Behaviors and Reduces the Level of Inflammatory Cytokines by Downregulating the Expression of TRAF6 in the Hippocampus of Rats Following Single-prolonged Stress
}

\author{
Yao Chen \\ China Medical University \\ Lei Tong \\ China Medical University \\ Peng-Yin Nie \\ China Medical University

\section{Yu-Lu Chen} \\ China Medical University \\ Lili Ji ( $\square$ llji@cmu.edu.cn ) \\ China Medical University
}

\section{Research}

Keywords: PTSD, neuroinflammation, microRNA-124, TRAF6, hippocampus

Posted Date: January 4th, 2022

DOI: https://doi.org/10.21203/rs.3.rs-1190728/v1

License: (c) (i) This work is licensed under a Creative Commons Attribution 4.0 International License. Read Full License 


\section{Abstract}

\section{Background}

MicroRNA-124-3p (miR-124) plays an important role in neuroprotective functions in various neurological disorders, but whether miR-124 participates in the pathological progression of posttraumatic stress disorder (PTSD) remains poorly understood.

\section{Methods}

In the present study, we evaluated the level of neuroinflammation in the hippocampus of rats exposed to single-prolonged stress (SPS) by western blot and immunofluorescence staining, while the effect of miR124 on PTSD-like behaviors was evaluated by behavioral test.

Results

Our results demonstrated that the level of miR-124 in the hippocampus of rats exposed to SPS was downregulated and that the upregulation of miR-124 could alleviate the PTSD-like behaviors of SPS rats. This effect of miR-124 might be achieved through TNF receptor-associated Factor 6 (TRAF6), which is a target gene of miR-124 and plays an important role in the immune and inflammatory reaction by regulating nuclear factor kappa-B (NF-KB). Furthermore, we found that miR-124 not only decreased the level of proinflammatory cytokines but also increased the expression levels of synaptic proteins (PSD95 and synapsin I) and regulated the morphology of neurons.

\section{Conclusion}

These results suggested that miR-124 might attenuate PTSD-like behaviors and decrease the level of proinflammatory cytokines by downregulating the expression of TRAF6 in the hippocampus of rats exposed to SPS.

\section{Introduction}

Posttraumatic disorder (PTSD), a severe psychiatric disorder that appears after exposure to lifethreatening or traumatic events, is characterized by symptoms of hyperarousal, intrusive reminders of the trauma, avoidance of trauma-related cues, and persistent negative alterations in cognition and mood [1, 2]. PTSD is highly associated with the development of chronic disease and other psychological syndromes [3-5], and it poses a significant burden on emotional and physical health and health care costs [6]. Some studies in recent years have suggested that immunological alterations might play a crucial role in the link between trauma and subsequent PTSD [7-9]. However, the specific mechanism needs to be further studied.

MicroRNAs (miRNAs), which are highly conserved 20-25 nucleotide endogenous small noncoding RNAs that regulate complementary mRNAs by modulating translation or degradation of mRNA [10], are 
involved in a series of biological processes, such as cell proliferation growth and differentiation, cellular metabolic activation and immune responses, in both physiological and pathological conditions [11-14]. Several miRNAs have been shown to play crucial roles in the regulation of central nervous system (CNS) development and regulate posttranscriptional gene expression in pathological cellular processes, including CNS injuries, psychiatric diseases and disorders [15-18]. Moreover, it has been shown that some miRNAs could exert immunomodulatory effects across the nervous system in both health and disease settings, as well as in the initiation and termination of inflammatory responses [19-22]. Many miRNAs are mainly expressed within the central nervous system (CNS), such as miR-9, miR-124, miR-132, and miR$101[23,24]$. MiR-124, one of the most enriched and best studied miRNAs that functions within the CNS and immune system, has been shown to play a regulatory role in some neurological diseases, such as Parkinson's disease, Alzheimer's disease, ischemic stroke, and psychiatric disorders [25-28]. MiR-124 can also regulate neuroinflammation in various CNS pathologies and might act as a novel therapeutic target [29-31]. Our previous study indicated that miR-142 downregulation could attenuate neuroinflammation in the hippocampus of rats with single-prolonged stress (SPS) [32]. However, whether other miRNAs play a role in regulating neuroinflammation in SPS rats still needs further study. We speculated that miR-124 might participate in the pathological process of PTSD through a certain regulatory mechanism.

Tumor necrosis factor receptor-associated factor (TRAF), a kind of multifunctional intracellular adaptin, integrates with different tumor necrosis factor (TNF) superfamilies and the toll-IL-1 receptor (TIR) superfamily, which mediates innate immunity [33]. TRAF6 is an important adaptin in the TRAF family and plays an important role in immune and inflammatory reactions [34]. TRAF6 can mediate the production of inflammatory mediators and interferon by participating in the activation of nuclear factor-kappa B (NF$\mathrm{KB}$ [ [35]. In addition, it has been reported that TRAF6 can mediate the generation of intracellular reactive oxygen species (ROS) [36]. TRAF6 is broadly expressed in mammalian tissues, including the brain, lung, liver, skeletal muscle and kidney, and is well conserved across species [34]. Accumulating evidence suggests that TRAF6 is related to central nervous system (CNS) diseases, such as stroke, traumatic brain injury, neurodegenerative diseases, neuropathic pain and CNS tumors [34, 37-39]. However, whether TRAF6 participates in the regulation of the pathological process of PTSD has not been studied. In addition, TRAF6 has been identified as a putative target gene of miR-124 via software analysis (http://www.targetscan.org). It would be interesting to know whether miR-124 contributes to regulating the neuroinflammation of PTSD by targeting the functions of TRAF6 and its downstream molecules.

In the present study, we found that the level of miR-124 was downregulated in the hippocampus of rats exposed to the SPS paradigm, while the level of TRAF6 was increased. Then, we showed that miR-124 could alleviate anxiety-like behaviors, depression-like behaviors, and memory impairment in SPS rats by injection of a lentivirus-delivered miR-124-3p through the lateral ventricle. Moreover, we showed that miR124 could reduce the levels of tumor necrosis factor (TNF)- $a$, interleukin (IL)- $1 \beta$ and IL-6, which were increased in SPS rats. Furthermore, we investigated the levels of PSD 95 and synapsin I to determine whether synaptic proteins were involved in the miR-124-regulated behaviors and memory deficits of SPS rats. 


\section{Methods}

\section{Animals}

Eight weeks-old adult healthy male Sprague-Dawley (SD) rats weighted about $200 \mathrm{~g}$ were provided by Liaoning Changsheng biotechnology co. (Liaoning, China). The rats were maintained with water and food freely available in the Experimental Animal Center of China Medical University. All experimental procedures on animals were approved by the Institutional Animal Care and Use Committee (China Medical University, China), which was in accordance with the National Institutes of Health Guide for the care and use of laboratory animals.

\section{Experimental groups and single-prolonged stress (SPS) procedure}

The animals were randomly divided into four groups, including control group, all animals were normal rats $(n=21)$; PTSD group, in which rats received SPS treatment $(n=21)$; miR-124-3p-mimic (miR-124) group, in which SPS rats were treated with miR-124-3p mimic virus $(n=21)$; miR-NC group, in which SPS rats were given scramble control lentivirus $(n=21)$. The rats then performed the following experimental procedures, including behavioral tests, immunofluorescence staining, and western blotting. The experimental paradigm is illustrated in Fig. $1 \mathrm{a}$.

The SPS procedure was used to establish rat model of PTSD, which was described by Liberzon et al previously [40]. First, rats were restrained for 2 hours in disposable restraint tubes, which were $150 \mathrm{~mm}$ in length and $58 \mathrm{~mm}$ in diameter. Following immobilization, rats were forced to swim for $20 \mathrm{~min}$ in a plexiglass cylinder filled with fresh water and the temperature of water was $24^{\circ} \mathrm{C}$. Then the animals were rested for $15 \mathrm{~min}$, and then exposed to ether vapor until they loss of consciousness. Upon awakening, animals were immediately returned to their home cages and left undisturbed for one week, and then they were subjected to behavioral tests. The SPS procedure was performed between 10 AM and 4 PM under bright white light conditions. For the controls, rats remained in their home cages and were undisturbed.

\section{High-throughput sequencing analysis of microRNAs related to SPS}

Library construction and sequencing analysis were carried out by Novogene (Beijing, China). Novogene analyses used ClusterProfiler software for enrichment analysis, including GO enrichment, DO enrichment, KEGG and Reactome database enrichment, to analyze and visualize the functional profiles of genomic coordinates, genes and gene clusters. Novogene performed differential expression analysis of two conditions/groups by using the DESeq2 R package. Clustering and grouping analyses used transcripts with FPKM values $>1$ and an adjusted $p$ value $<0.05$.

\section{Injection of the lentiviral-mediated miR-124-3p mimics}

The rats were fixed in a brain stereotaxic apparatus after anesthetized with pentobarbital sodium. Afterwards, ten microliters of the miR-124-3p mimic (LV-miR-124; Wanlei Biotechnology Co., Ltd.; Shenyang, China) and mimic-NC lentivirus (LV-NC; Wanlei Biotechnology), whose titer was $1 \times 10^{8}$ 
transducing $\mathrm{U} / \mathrm{ml}$ for the experiments, was injected into the right lateral ventricle of rats and the coordinates were as follows: $1.2 \mathrm{~mm}$ posterior to the bregma, $1.8-2.0 \mathrm{~mm}$ right to sagittal suture, and 4.0 $\mathrm{mm}$ below the external surface. The transfection efficiency was evaluated by RT-qPCR 7 days after injection.

\section{Behavioral test}

All behavioral tests were conducted at daytime in a sound-isolated room. It was necessary to make rats acclimated in the laboratory for $2 \mathrm{~h}$ before starting the test. Six rats per group were subjected to each of the experimental methods including open field test (OFT), elevated plus maze (EPM) test, forced swimming test (FST), and Fear Conditioning Test (FCT), allowing animals to rest for at least half an hour between each stage.

\section{Open Field Test}

OFT was used to evaluate anxiety-like behavior in rats. Animals were placed into the same corner of the apparatus, which was an enclosed square bottom made of dark opaque plexiglas $(720 \mathrm{~mm} \times 720 \mathrm{~mm})$ surrounded by walls $(400 \mathrm{~mm}$ high) respectively. All rats were placed facing the same direction and all locomotor activities in 5 minutes were recorded by a video camera and the behavioral parameters were then collected by the SuperMaze software (Shanghai Xinruan Information Tech Co., Shanghai, China). The measurements recorded including: the total travel distance and duration in the center area (the four squares in the center of apparatus) and total immobility time. The floor surface was wiped clean thoroughly using $70 \%$ ethanol between every two animal experiments to eliminate the influence of animal odor on the results of the experiment.

\section{Elevated plus maze (EPM) test}

The EPM test was also used to evaluate anxiety-like behavior after OFT in the present study. The EPM apparatus, which was elevated $50 \mathrm{~cm}$ above the floor, was consisted of a central platform $(10 \times 10 \mathrm{~cm})$,

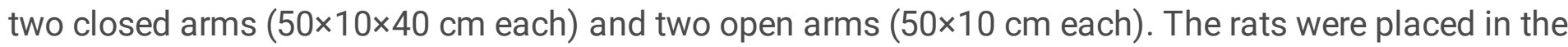
central platform facing an open arm respectively, and all locomotor activities were recorded by a video camera during the 5 mins test. The following parameters was recorded: the percentage of time spent in the open arms, the percentage of entries into the open arms. The maze was cleaned using $70 \%$ ethanol after each subject had been tested.

\section{Forced swim test (FST)}

The FST was used for assessing depression-like behavior in rats. Briefly, the animals were respectively placed in and swam for $6 \mathrm{~min}$ in the apparatus filled with water, whose height was $30 \mathrm{~cm}$ and the temperature was $23-25^{\circ} \mathrm{C}$. The total amount of immobile time of each rat was recorded. Animals were immediately removed from the cylinder and settled in a clean and dry cage after testing. Water was renewed to avoid bias between each test. 


\section{Fear Conditioning Test (FCT)}

The conditioning chamber (XR-XC404; Shanghai Xinruan Information Tech Co., Ltd.) the length, width and height of which are $320 \mathrm{~mm}, 280 \mathrm{~mm}$, and $500 \mathrm{~mm}$ respectively consists of four vertical Plexiglas sides. The floor of the condition chamber was consisted of horizontal metal bars $(0.5-\mathrm{cm}$ diameter, spaced 1.5 $\mathrm{cm}$ apart) connected to an electric shock generator and the animals were allowed to explore for 3 minutes on the first day of fear conditioning. After that, rats were presented with a $30-\mathrm{s}$ tone $(75 \mathrm{~dB}, 3 \mathrm{kHz})$, followed immediately by a 2-s footshock $(0.8 \mathrm{~mA})$ and this process was repeated for 3 times and the inter-trial intervals was 30 s. One day after training animals were placed in the same chamber and observed for 6 min without being shocked and scored for the freezing behavior. For the auditory-cued fear test, the animals were placed in another chamber for a $3 \mathrm{~min}$. Then, activate the tone for another $3 \mathrm{~min}$ and score the freezing behavior. The definition of freezing behavior, which was recorded as a a percentage of the observation period, was the absence of any visible movement other than breathing.

\section{Cell culture and transfection}

The PC12 cell line, which can differentiate into neuron-like cells, was induced by the medium with 100 $\mathrm{ng} / \mathrm{mL}$ nerve growth factor (NGF, R\&D, Minneapolis, MN, USA) and used to establish neuronal models. Cells were cultured in Dulbecco's modified Eagle medium (DMEM, Gibco) containing 10\% fetal bovine serum (FBS, Gibco), and $1 \%$ penicillin/streptomycin (P/S) in an incubator with a temperature of $37^{\circ} \mathrm{C}$, a humidified atmosphere with $5 \% \mathrm{CO}_{2}$. PC12 cells were incubated for 12 hours using serum-free DMEM before differentiation. Then cells were induced by NGF for 24 hours and the neuron-like cells were used for follow-up experiments. Additionally, human embryonic kidney 293T (HEK293T) cells for dual luciferase reporter assay were cultured in high glucose DMEM containing $10 \% \mathrm{FBS}$ and $1 \% \mathrm{P} / \mathrm{S}$.

\section{Immunofluorescence staining}

Rats were anesthetized by pentobarbital sodium $(50 \mathrm{mg} / \mathrm{kg})$ through intraperitoneal injection and then perfused with saline and fixed by $4 \%$ paraformaldehyde (PFA) dissolved in phosphate buffer saline (PBS). The brains were removed gently and fixed with $4 \%$ PFA at $4{ }^{\circ} \mathrm{C}$ overnight. The brain slices of $7 \mu \mathrm{m}$ thick were obtained by a cryostat after dehydrating with gradient $(15 \%, 25 \%, 30 \%)$ sugar solution in PBS. For double immunofluorescence, the sections were permeabilized using $0.2 \%$ Triton X-100 in PBS for 10 min at room temperature after washing 3 times with PBS. Then the sections were blocked by $5 \%$ bovine serum albumin (BSA) for $2 \mathrm{~h}$ at room temperature. After that, the brain sections were incubated with the following primary antibodies (all were obtained from Abcam) at $4{ }^{\circ} \mathrm{C}$ overnight: mouse $\beta$-tubulin III primary antibody (1: 500), rabbit TRAF6 primary antibody (1:300), rabbit IL-1 $\beta$ primary antibody (1: 300), rabbit IL-10 primary antibody (1:300), rabbit iNOS primary antibody (1:300), rabbit PSD 95 primary antibody (1:200), and goat synapsin I primary antibody (1:200). The brain sections were incubated in goat anti-mouse 488-conjugated secondary anti-body (1: 600, Abcam) or donkey anti-rabbit 594conjugated secondary antibody (1: 600, Abcam) for $2 \mathrm{~h}$ at $37^{\circ} \mathrm{C}$ after washing 3 times using PBS. 4,6diamidino-2-phenylindole (DAPI) was used to stain the nuclei and slides were cover slipped with Antifade 
Mounting Medium (Beyotime Biotech, Shanghai, China). Images were captured under a fluorescence microscope using standard laser lines and filters.

\section{Western blotting analysis}

The hippocampal tissues were stripped individually from six rats in each group and lysed with radio immunoprecipitation assay buffer (RIPA, Beyotime Biotech, Shanghai, China) containing Phenylmethanesulfonyl fluoride (PMSF, Beyotime, China). The extracted proteins were sonicated and incubated overnight at $4{ }^{\circ} \mathrm{C}$ and then centrifuged at $12000 \mathrm{rpm}, 4^{\circ} \mathrm{C}$ for $30 \mathrm{~min}$. The supernatants were collected and the concentration of protein was determined by the Bicinchoninic acid (BCA) Protein Assay Kit (Beyotime, China); Equal amounts of samples $(30 \mu \mathrm{g})$ was added to the wells and separated by SDSPAGE gel and then the proteins were transferred onto polyvinylidene fluoride (PVDF) membrane (Millipores, $0.45 \mu \mathrm{m}$, Billerica, MA, USA). After blocking with with $5 \% \mathrm{BSA}$ at $37^{\circ} \mathrm{C}$ for $1 \mathrm{~h}$, the membranes were incubated with the following primary antibodies: TRAF6, IL-1ß, IL-10, IL-6, TNF-a, iNOS, PSD95, Synapsin I, and NF-KB p65 (all diluted to 1:1000 and purchased from Abcam) and GAPDH (1:10000, Proteintech Biotech, Wuhan, China) at $4{ }^{\circ} \mathrm{C}$ overnight. The membranes were then incubated by the horseradish peroxidase-conjugated secondary antibody (1:10000, Proteintech) at $37^{\circ} \mathrm{C}$ for $1 \mathrm{~h}$. Enhanced chemiluminescence (ECL; Uscn Life, China) was used to detect the protein bands on a Chemi-Doc XRS + imaging system (Bio-Rad, USA). The gray value of all proteins was quantified by ImageJ software (NIH).

\section{Golgi staining and spine morphology analysis}

After behavioral tests, rats were deeply anesthetized with sodium pentobarbital, and the brains were removed and fixed in a specified formulated solution. We replaced it regularly and then stained it with the Rapid Golgi protocol using the Hito Golgi-Cox OptimStain Kit (Hitobiotec Inc, Kingsport, TN, USA) according to the methods provided by the manufacturer. Brain sections, which were $100-\mu \mathrm{m}$ thick and sectioned using a cryostat, were collected on gelatinized glass slides and then dehydrated in absolute ethanol. The sections were coverslipped using undiluted xylene-based resinous mounting medium after being cleared in xylene. The sections were placed under an Olympus light microscope and imaged using software with a 100NA 1.4 oil immersion lens. The dendrites of hippocampal pyramidal neurons were randomly selected for imaging, and the dendritic spine density was calculated. Dendritic spine density was the number of spines per $10 \mu \mathrm{m}$ of dendritic length from somatic cells.

\section{Transmission electron microscopy (TEM) observations}

Ultrastructural alterations were also detected using TEM. Briefly, precold heparinized $0.9 \%$ saline and 0.01 M PBS (pH 7.2-7.4) containing $2 \%$ paraformaldehyde and $2.5 \%$ glutaraldehyde was used for perfusion. The hippocampal tissue of each rat $\left(1 \mathrm{~mm}^{3}\right)$ was removed and then fixed for $24 \mathrm{~h}$ at $4{ }^{\circ} \mathrm{C}$, followed by $2 \mathrm{~h}$ of postfixation with $1 \%$ osmium tetroxide. Ultrathin sections were stained with uranyl acetate and lead citrate. All grids were detected under a transmission electron microscope ( $\mathrm{H}-7650$, Hitachi Limited, Japan) operating at $80 \mathrm{kV}$. 


\section{Real-time quantitative PCR (RT-qPCR)}

The isolation kit (Vazyme Biotech, Nanjing, China) was used to extract total RNA from rat hippocampus and the miRNA first strand cDNA synthesis kit (Vazyme Biotech) was used to reversely transcribe the extracted RNA into CDNA. Real time PCR reaction were performed with specific primers (GenePharma, Shanghai, China) using the StepOnePlus system (Applied Biosystems, USA). The primer sequences used for analysis were listed as following: miR-124-3p, Forward, 5'-TGTCTCTAAGGCACGCGGT-3' and Reverse, 5'-TATGGTTGTTCACGACTCCTTCAC-3'; TRAF6: Forward, 5'-CGCCAAAATGGAAACGCAGA-3' and Reverse, 5'-TGCTTCCATCTCGGCAACTT-3'; U6: Forward, 5'-CGCTTCGGCAGCACATATAC -3' and Reverse, 5'TTCACGAATTTGCGTGTCATC-3'; ACTIN: Forward, 5'-ACATCCGTAAAGACCTCTATGCCAACA -3' and Reverse, 5'-GTGCTAGGAGCCAGGGCAGTAATCT-3'. Relative gene fold changes relative to control was analyzed using the The $2^{-\triangle \triangle \mathrm{Ct}}$ method.

\section{Dual luciferase reporter assay}

A luciferase reporter assay was performed to detect whether miR-124-3p could bind to TRAF6 mRNA. The wild-type TRAF6 3'UTR reporter plasmid (pmiR-TRAF6-wt) containing the putative binding sites of miR124 and mutant TRAF6 3'UTR reporter plasmid (pmiR-TRAF6-mut) were constructed by GenePharma (Shanghai, China). The reporter vectors containing pmiR-TRAF6-wt or pmiR-TRAF6-mut were cotransfected with miR-124 mimics or scrambled RNA into HEK293T cells using Lipofectamine 2000 (Invitrogen, USA) according to the manufacturer's instructions. Luciferase activities were measured by a dual-luciferase reporter assay system (Promega, USA) in a microplate reader according to the manufacturer's information. The relative luciferase activity was evaluated by the ratio of Renilla luciferase activity to firefly luciferase activity and expressed as a percentage of the control.

\section{Fluorescence in situ hybridization (FISH)}

FISH was used to detect the colocalization of miR-124 and TRAF6 in the hippocampal cells of rats. The frozen slices were treated with Proteinase $\mathrm{K}$ digestion at $37^{\circ} \mathrm{C}$ for 20 min after rehydration in citrate buffer. Then, the brain slices were immersed in RNase-free water for 3 min and hydrated in tap water with a gradient series of alcohol. The slices were incubated in hybridization buffer supplemented with denatured 5' CY3-labeled locked nucleic acid (LNA) probes directed against miR-124-3p and 5' FAMlabeled locked nucleic acid (LNA) probes directed against TRAF6 (GenePharma, China) overnight at 37 ${ }^{\circ} \mathrm{C}$. After washing, the sections were counterstained with DAPI for $20 \mathrm{~min}$ and observed under a confocal microscope.

\section{Statistical analysis}

All data were analyzed with SPSS19.0 statistical software, and the measured data were expressed as mean \pm SEM (standard error of mean). Statistical comparisons between groups were performed by independent sample test. One-way or two-way analysis of variance (ANOVA) and Bonferroni's post hoc 
test analysis were used to analyze the data between the groups. In all cases, $P<0.05$ was considered a significant difference.

\section{Results}

\section{The level of miR-124 or TRAF6 was changed in the hippocampus of rats exposed to SPS}

To examine the differentially expressed microRNAs in the hippocampus between rats 7 days after SPS and normal rats (control), we screened the microRNA expression patterns with sequencing analysis. The results showed that the level of miR-124 in the hippocampus of SPS rats was decreased significantly ( $t=$ 2.255, $P=0.0436$, Fig. 1b and 1c). We further verified the decreased level of miR-124 by RT-qPCR analysis ( $t=6.521, P=0.0029$, Fig. $1 \mathrm{~d})$. These results suggested that miR-124 might be involved in the pathogenesis of PTSD.

To explore the potential molecular mechanisms of miR-124 in the pathological process of PTSD, we used software analysis to identify downstream proteins related to it. The results showed that miR-124-3p might have a binding site in the 3'-UTR of TRAF6. Furthermore, we tested the expression levels of TRAF6 in the hippocampus of SPS rats by Western blotting, and the results showed that the level was increased significantly ( $t=6.186, P=0.0001$, Fig. $1 \mathrm{e}$ and $1 \mathrm{f})$. This result suggested that miR-124 might participate in the pathogenesis of PTSD through TRAF6.

To further study the specific mechanism of miR-124 in PTSD, we injected a miR-124-3p mimic delivered by the lentivirus into the right lateral ventricle in rats exposed to SPS. GFP fluorescence was observed in the hippocampus $7 \mathrm{~d}$ after administration, indicating successful transfection (Fig. 1g). The level of miR124 in the hippocampus was also tested by RT-qPCR, and the results showed that it was significantly increased in the miR-124 group compared with the miR-NC group ( $t=5.308, P=0.0061$, Fig. $1 \mathrm{~h})$.

\section{miR-124 alleviated PTSD-like behavior in SPS rats}

To explore whether miR-124 could affect PTSD-like behaviors in rats exposed to SPS, different behavioral tests, including the OFT, EPM, FST and FCS test, were used to detect depressive-like behavior, anxiety-like behavior and memory abnormalities. In the OFT, we found that there was no significant difference in the total distance traveled among the four groups (Fig. 2b). However, rats in the SPS group traveled a significantly shorter distance and spent less time in the central area than the rats in the control group. The administration of miR-124 significantly reversed this trend $(F(3,20)=6.509, P=0.0030$, Fig. 2c; $F(3$, $20)=29.88, P<0.0001$, Fig. $2 \mathrm{~d}$ ). The EPM results showed that SPS rats spent significantly less time on the open arms and fewer entries into the open arms than the control group. However, this trend was reversed by miR-124 injection $(F(3,20)=22.30, P<0.0001$, Fig. 2f; $F(3,20)=36.36, P<0.0001$, Fig. $2 \mathrm{~g})$. Furthermore, the FST results showed that the immobility time of the rats exposed to SPS was longer than that of the rats in the control group, whereas rats in the miR-124 group had a shorter immobility time than those in the SPS group $(F(3,20)=9.855, P=0.0003$, Fig. $2 \mathrm{~h})$. Finally, the rats were subjected to FCS to determine whether miR-124 had an effect on spatial learning and memory impairment in SPS rats. The 
results showed that rats in the SPS group displayed enhanced fear learning behavior compared to the control groups, and miR-124 administration reversed this trend (context: $F(3,20)=9.242, P=0.0005$, Fig. 2i; tone: $F(3,20)=15.74, P<0.0001$, Fig. $2 \mathrm{j})$.

Based on these results, the rats exposed to the SPS procedure showed obvious anxiety-like behaviors and depression-like behaviors and enhanced fear learning memory, and miR-124 injection significantly alleviated these behaviors.

\section{MiR-124 decreased the levels of TRAF6 and NF-KB in the hippocampus of SPS rats}

Inflammation is an important molecular mechanism in the pathogenesis of PTSD, and TRAF6 plays a pivotal role in regulating immune and inflammatory responses by mediating activation of the NF-KB signaling pathway [41]. We next investigated the levels of TRAF6 and NF-KB associated with inflammation in the hippocampus of SPS rats through immunofluorescence staining and Western blotting. Immunofluorescence staining assays indicated that miR-124 markedly attenuated the expression of TRAF6 in the hippocampus of rats exposed to SPS (Fig. 3a). Western blotting analysis was also used to detect the expression level of TRAF6 in each group, and the results revealed that miR-124 significantly decreased the expression levels of TRAF6, which was upregulated in the hippocampus of rats in the SPS group compared to that in the control group $(F(3,20)=6.660, P=0.0027$, Fig. $3 \mathrm{~b}$ and $\mathrm{c})$.

The levels of NF-KB p65 were significantly higher in the hippocampus of rats in the SPS group than in the control group, but this trend could be significantly reversed by miR-124 $(F(3,20)=7.164, P=0.0019$, Fig. 3d and f).

These results further verified that the levels of TRAF6 and NF-KB were upregulated in the hippocampus of rats exposed to the SPS procedure and that these trends could be reversed by miR-124 injection.

\section{MiR-124 could regulate the level of inflammatory cytokines in the hippocampus of rats exposed to the SPS procedure}

To detect whether miR-124 affects the inflammatory response in the hippocampus of SPS rats, we further detected the levels of proinflammatory cytokines (IL-1 $\beta$, TNF- $a$, and IL-6) and anti-inflammatory cytokines (IL-10) in the hippocampus of SPS rats by immunofluorescence staining and Western blotting. The results of immunofluorescence staining showed that the expression level of IL-1 $\beta$ was increased in the hippocampus of rats exposed to SPS, while miR-124 reversed this trend (Fig. $4 \mathrm{a}$ ). The results are shown in Fig. 4. Compared to the control group, the levels of TNF-a, IL-1 $\beta$, and IL- 6 expression were significantly increased in the hippocampus of rats exposed to the SPS procedure, while miR-124 could reverse these trends significantly (TNF-a: $F(3,20)=11.41, P=0.0001$, Fig. 4c and d; IL-1 $\beta: F(3,20)=7.787, P=0.0012$, Fig. $4 \mathrm{c}$ and e; IL-6: $F(3,20)=7.048, P=0.0020$, Fig. $4 \mathrm{c}$ and f). In contrast, the level of IL-10 was significantly decreased in the hippocampus of rats in the SPS group, while this trend was also reversed by miR-124 $(F(3,20)=9.800, P=0.0003$, Fig. $4 \mathrm{~g})$. The results of immunofluorescence staining for IL-10 were consistent with the Western blotting results (Fig. $4 \mathrm{~b})$. 
Nitric oxide (NO), the first gaseous neurotransmitter discovered in the brain, is synthesized from l-arginine by enzymes called NO synthases (NOSs), including neuronal NOS (nNOS), inducible NOS (iNOS) and endothelial NOS (eNOS) [42, 43]. NO not only regulates nerve proliferation, survival and differentiation but also regulates different aspects of neurotransmitter release and synaptic dynamics [44, 45]. In the present study, we also explored the level of iNOS, and the results of immunofluorescence staining indicated that the iNOS-positive cells were increased in the hippocampus of rats in the SPS group, but the trend was reversed by treatment with miR-124 (Fig. 5a). The results showed that the expression level of iNOS was significantly increased in the hippocampus of SPS rats, but this trend could be abolished by the injection of $\operatorname{miR}-124(F(3,20)=11.27, P=0.0002$; Fig. 5b and $c)$.

These results indicated that treatment with the miR-124 mimic might rescue the inflammatory response in the hippocampus of rats exposed to the SPS procedure.

\section{MiR-124 upregulated the expression level of synaptic proteins in the hippocampus of rats exposed to SPS}

Pre- and post-synaptic proteins play an important role in the pathobiology of various psychiatric disorders, especially regulating cognitive function in PTSD. Postsynaptic density protein 95 (PSD-95), a member of the membrane-associated guanylate kinases (MAGUKs), is a class of scaffold proteins that play essential roles in excitatory synaptic transmission and organization of dendritic spines [46].

Synapsin I is a neuron-specific cytosolic phosphoprotein that can regulate the kinetics of synaptic vesicle fusion and the function of the synaptic response to extracellular signals [47]. To explore whether the miR124 mimic could affect neuronal morphology and function, we further evaluated the expression levels of PSD-95 and synapsin I in the hippocampus of rats in each group. Immunofluorescence staining showed that the intensities of both PSD95 and synapsin I decreased in $\beta$-tubulin III-positive cells in the hippocampus of rats in the SPS group, but the trend was reversed by treatment with miR-124 (Fig. 6a and b). The Western blotting results showed that there were significantly lower levels of PSD95 and synapsin I proteins in the hippocampus of the SPS group than in the hippocampus of the control group. In contrast, miR-124 overexpression abrogated the downregulation of these two proteins (PSD95: $F(3,20)=7.584, P$ $=0.0014$, Fig. $6 \mathrm{c}$ and d; synapsin I: $F(3,20)=8.185, P=0.0009$, Fig. $6 \mathrm{c}$ and $\mathrm{e})$.

These results indicated that miR-124 might affect synaptic morphology and further affect neural function by regulating synaptic protein expression in the hippocampus of rats exposed to SPS.

\section{miR-124 upregulated the mature spine in the hippocampus of rats exposed to SPS}

Dendritic spines, which are small membrane protrusions on dendrites, play an important role in synaptic formation and neural circuits. To detect whether miR-124 affects synaptic formation, we next tested the morphology and dendritic spine density of hippocampal neurons in the control, SPS and miR-124 groups through Golgi-Cox staining. Compared with the control group, the rats in the SPS group exhibited a significant reduction in spine density in the hippocampus, but the injection of miR-124 prevented the SPSinduced reduction in spine density $(F(2,6)=17.74, P=0.0030$, Fig. 7a-e). Furthermore, we measured the percentage of four types (filopodia, thin, stubby and mushroom) of dendritic spines according to their 
sizes and shape. The results showed that the proportion of stubby and mushroom spines in the SPS group was lower than that in the control group, and this trend was reversed in the miR-124 group. For filopodia spines, the spine density was significantly increased in the hippocampus of rats in the SPS group, while this trend was also reversed by miR-124 (stubby: $F(2,6)=22.94, P=0.0015$; mushroom: $F(2,6)=8.410, P=0.0182$; filopodia: $F(2,6)=13.62, P=0.0059$; Fig. $7 \mathrm{f})$.

Our results indicated that the proportion of mature dendritic spines in the hippocampal neurons of rats exposed to SPS was significantly decreased, while miR-124 reversed this trend. This result suggested that miR-124 might regulate the morphology of hippocampal neurons directly or indirectly and that this effect might be involved in the pathological process of PTSD.

\section{The effect of miR-124 on ultrastructural alterations in the hippocampus of rats exposed to SPS}

Synaptic structures and function have been implicated in the progression of learning and memory [48]. Therefore, we sought to detect whether miR-124 could affect the ultrastructure of hippocampal synapses in rats exposed to SPS by TEM. As shown in Figure 8a, the presynaptic and postsynaptic membranes were clear and had complete outlines. The synaptic cleft was clearly discernible, and abundant PSD was observed in the cells of the control group. However, the structural parameters of the synaptic junction were altered in the hippocampal cells of rats in the SPS group, with a widened synaptic cleft and a thinned PSD. Quantitatively, in the SPS group, the width of the synaptic cleft was remarkably increased, and the PSD thickness was significantly thinner than that in the control group. However, these changes in the width of the synaptic cleft and PSD thickness were all significantly reversed by miR-124 treatment (thickness of PSD: $F(2,6)=10.97, P=0.0099$, Fig. 8b; width of synaptic cleft: $F(2,6)=13.54, P=0.0060$, Fig. 8c).

These results showed that the ultrastructure of hippocampal neurons was altered by the SPS procedure, while miR-124 injection could significantly repair it. This might be involved in the mechanism by which miR-124 can alleviate behavioral abnormalities in SPS rats.

\section{MiR-124 directly targets the TRAF6 3'UTR and regulates NF-KB}

As mentioned above, TRAF6 is a target of miR-124 predicted by bioinformatics analysis, and microRNAs could inhibit gene expression by binding to the 3 '-UTR site of target genes. To verify whether TRAF6 is one of the predicted target genes of miR-124-3p, we further studied the relationship between miR-124 and TRAF6 by the dual-luciferase reporter assay. The bioinformatic database (miRDB and TargetScan) suggested that there are two potential binding sites between miR-124-3p and the 3'-UTR of TRAF6, which contained conserved seed sequences at positions 47-53 and 341-348 (Fig. 9a). We constructed TRAF6 3'UTR (wild-type) and mutated TRAF6 3'-UTR (mutant) luciferase reporter plasmids and transfected them into 293T cells alongside miR-124 mimic or scrambled oligonucleotides. The results demonstrated a significant decrease in relative luciferase activity in HEK 293 T cells cotransfected with wild-type vectors and the miR-124 mimic $(F(3,20)=5.402, P=0.0069$, Fig. 9b), but downregulation was not observed in the other groups. In addition, a FISH assay was further performed, and the results revealed a clear overlap 
between miR-124 and TRAF6 in hippocampal tissues (Fig. 9e). Taken together, these results indicated that miR-124 might bind to the TRAF6 3'-UTR directly.

To further verify the regulation between miR-124 and TRAF6 expression levels, neuron-like cells were transfected with LV-miR-124 or LV-miR-124 scramble. The expression level of TRAF6 was tested by Western blotting analysis, and the results are shown in Fig. $8 \mathrm{c}$ and d. Compared with the miR-NC group transfected with the LV-miR-124 scramble, the TRAF6 protein levels in the cells overexpressing miR-124 were significantly reduced ( $t=4.308, P=0.0015$, Fig. $9 \mathrm{c}$ and d). To investigate whether NF-KB p65 was modulated by TRAF6, we evaluated the expression level of NF-KB p65 in neuron-like cells transfected with siRNA-TRAF6 or siRNA-NC. Compared with the control group, the expression level of NF-kB p65 in the siTRAF6 group was significantly reduced $(F(2,15)=6.781, P=0.0080$, Fig. $9 f$ and $9 \mathrm{~g})$.

These findings indicate that TRAF6 was a direct target of miR-124 and that overexpression of miR-124 could suppress the protein expression levels of TRAF6 protein, which could regulate NF-KB. MiR-124 might regulate the level of NF-KB by downregulating the expression level of TRAF6.

\section{Discussion}

In this study, the level of miR-124 was decreased in the hippocampus of rats exposed to the SPS procedure and the injection of miR-124 through the lateral ventricle could alleviate PTSD-like behaviors, including anxiety, depression and memory impairment, in SPS rats, indicating an important role for miR124 in regulating abnormal behaviors during the pathological process of PTSD. TRAF6, a predicted target gene of miR-124 that was identified by the dual-luciferase reporter assay and FISH assay, was upregulated in the hippocampus of rats exposed to SPS. These factors might be novel targets for the treatment or prevention of PTSD; thus, we further explored the possible mechanism by which they regulate abnormal behaviors.

It has been shown that the levels of proinflammatory cytokines, including TNF- $\alpha$, IL-1 $\beta$, and IL-6, were increased in the hippocampus of rats exposed to the SPS procedure [32] and could be decreased by miR124 , as shown by the results in our study. This result indicated that miR-124 might reduce the inflammatory response of the central nervous system, which is characterized by the activation of glial cells that release a series of soluble factors, including cytokines, reactive oxygen species (ROS), reactive nitrogen species (RNS) and lipid metabolites $[49,50]$. Microglia may shape the activity of neurons, and the action of microglia may be influenced by neurons in the central nervous system (CNS) or in the peripheral nervous system [51-53]. Growing evidence indicates that miR-124 maintains the quiescence of microglia and plays a key role in the regulation of signaling molecules underlying synaptic plasticity and memory [17, 20,54-56]. The results of the present study showed that miR-124 could downregulate the activity of microglia, which might be caused by neuroinflammation that might be induced by the abnormal morphology of neurons in the hippocampus of rats exposed to the SPS procedure. We further found that the promoted neuronal inflammation might affect the expression level of synaptic proteins, such as PSD95 or synapsin I, in the hippocampal neurons of rats exposed to the SPS procedure, and this 
might be related to the synaptic morphology and further affect neural function, whose impairment might participate in the pathological process of rats' abnormal behaviors induced by SPS. However, the mechanism of miR-124 on the interaction between microglia and neurons in the hippocampus of SPS rats has not yet been studied.

MiRNAs regulate multiple target gene mRNAs mainly by inhibiting target gene translation, inhibiting combining, or cutting off the target gene's RNA molecule and are involved in a variety of physiological processes $[28,57]$. MiR-124-3p is considered a brain-specific miRNA that is mainly expressed in neuronal cells and best studied in functions within the nervous system and immune system [26]. Several studies have shown that the dysregulation of miR-124 expression in the brain might contribute to pathogenic changes in neurodegenerative diseases, such as Alzheimer's disease (AD), Parkinson's disease (PD), and Huntington's disease (HD) [58-63]. MiR-124 has also been identified as a neuroprotective factor in promoting neurogenesis during brain development and maintaining synaptic homeostasis in the adult brain $[64,65]$. It has been reported that miR-124 can regulate multiple molecules in TLR4 signaling, such as TLR4, MyD88 and TRAF6 [66]. Our present study indicates that miR-124 might be involved in pathogenesis and that its dysregulation might affect neuronal cells through binding to TRAF6, a target gene of miR-124. TRAF6, which is an essential intermediate in the NF-KB signaling pathway, is involved in the process of inflammation [35,67], is closely related to PSD-95 and contributes to synapse assembly, function and plasticity [68]. It has also been proven that TRAF6 could interact with the synaptic cell adhesion molecule neuroplastin during neuronal development [69]. Our results suggest that SPS-induced downregulation of miR-124 was involved in increased neuroimmune signaling pathway mediated by TRAF6, which might further increase the expression of inflammatory factors, such as TNF- $\alpha$ and IL-1 $\beta$, in the hippocampus of rats exposed to SPS.

$\mathrm{NF}-\mathrm{KB}$, an important nuclear transcription factor, plays an important role in regulating the immune response to infection by controlling the expression of inflammatory genes and the production of cytokines, which can result in inflammatory disorders [70-72]. In the present study, we focused on exploring whether miR-124 can regulate the activation of NF-KB by targeting TRAF6 in the hippocampus of rats exposed to SPS. The results showed that miR-124 could suppress the expression level of NF-KB in the hippocampus of SPS rats in vivo and that downregulation of TRAF6 could significantly decrease the expression level of NF-KB in vitro. Meanwhile, the levels of proinflammatory cytokines, including TNF-a, IL-1 $\beta$, IL- 6 and inducible nitric oxide synthase (iNOS), were decreased, and the levels of anti-inflammatory cytokines, such as IL-10, were increased by miR-124. Thus, we suggest that miR-124 might alleviate the neuroinflammatory response in the hippocampus induced by SPS by downregulating the activation of NF-KB by combining with TRAF6.

Previous studies have shown that proinflammatory cytokines might influence neuroplasticity and neuronal function [73,74], which was also observed in the present study. Dendritic spines are structural and functional specializations of neurons at the point of entry of excitatory synaptic stimulation to postsynaptic neurons $[75,76]$. The postsynaptic density of dendritic spines includes a variety of proteins associated with actin filaments, and the interaction among these proteins is the basis of the plasticity of 
spines under both normal and pathological conditions [77]. Our study showed that the density of dendritic spines in hippocampal neurons and the expression level of synaptic proteins, including PSD95 and synapsin I, were affected by the SPS procedure and that miR-124 could significantly reverse this abnormality. This result indicated that neuroinflammation induced by SPS might change the structure of synapses and affect the expression level of synaptic proteins, which might further cause abnormal behaviors in animals and aggravate neuroinflammation through interactions between neurons and microglia.

\section{Conclusions}

In summary, we showed that the level of miR-124 in the hippocampus was decreased and that the expression level of its target TRAF6 was concurrently increased. Furthermore, the present research provided a possible mechanism by which miR-124 could alleviate the PTSD-like behaviors of rats exposed to SPS by regulating neural morphology and neuroinflammation through the NF-KB signaling pathway by targeting TRAF6. However, other detailed signaling cascades involved in the regulation of attenuating PTSD-like behaviors of rats exposed to a SPS procedure by miR-124 need to be explored in further experimental research. These works will provide evidence that miR-124 or TRAF6 might be a potential target for the prevention or treatment of PTSD in the future.

\section{Abbreviations}

AD: Alzheimer's disease; BCA: Bicinchoninic acid; BSA: bovine serum albumin; CNS: central nervous system; DAPI: 4,6-diamidino-2-phenylindole; EPM: elevated plus maze; ECL: Enhanced chemiluminescence; eNOS: endothelial NOS; FST: forced swimming test; FCT: Fear Conditioning Test; FISH: Fluorescence in situ hybridization; HEK293T: human embryonic kidney 293T; HD: Huntington's disease; iNOS: inducible NOS; MicroRNAs: miRNAs; MAGUKs: membrane-associated guanylate kinases; NF-kB: nuclear factor kappa-B; NO: Nitric oxide; NOSs: NO synthases; nNOS: neuronal NOS; OFT: open field test; PTSD: Posttraumatic stress disorder; PD: Parkinson's disease; PMSF: Phenylmethanesulfonyl fluoride; PFA: paraformaldehyde; PBS: phosphate buffer saline; PVDF: polyvinylidene fluoride; PSD-95: Postsynaptic density protein 95; pmiR-TRAF6-wt: The wild-type TRAF6 3'UTR reporter plasmid; pmiRTRAF6-mut: the mutant TRAF6 3'UTR reporter plasmid; RIPA: radio immunoprecipitation assay buffer; ROS: reactive oxygen species; RNS: reactive nitrogen species; RT-qPCR: Real-time quantitative PCR; SD: Sprague-Dawley; SPS: Single-prolonged stress; TRAF6: Tumor necrosis factor receptor-associated factor 6; TEM: Transmission electron microscopy;

\section{Declarations}

\section{Ethical Approval and Consent to participate}

All experimental procedures on animals were approved by the Institutional Animal Care and Use Committee (China Medical University, China), which was in accordance with the National Institutes of 
Health Guide for the care and use of laboratory animals.

\section{Consent for publication}

Not applicable.

\section{Availability of data and materials}

Please contact the author for data requests.

\section{Competing interests}

The authors declare that they have no competing interests.

\section{Funding}

This work is supported by the National Natural Science Foundation of China (31900850 to Ji L).

\section{Authors' contributions}

This work was primarily conceived by LJ and YC; data was collected by YC, PN, YC and analyzed by YC, LT and LJ. Manuscript was written by YC, LT, YC, and LJ. Figures were produced by YC. All authors read and approved the final manuscript.

\section{Acknowledgements}

None

\section{Authors' information}

Department of Anatomy, College of Basic Medical Sciences, China Medical University, No.77 Puhe road, Shenyang North New Area, Shenyang, Liaoning Province, P.R. China.

\section{References}

1. Bisson, J.I., et al., Post-traumatic stress disorder. BMJ, 2015. 351: p. h6161.

2. American Psychiatric Association.Diagnostic and Statistical Manual of Mental Disorders, 5th edn. American Psychiatric Publishing,Arlington,VA, 2013.

3. Edmondson, D., et al., Posttraumatic stress disorder and risk for coronary heart disease: a metaanalytic review. Am Heart J, 2013. 166(5): p. 806-14.

4. Afari, N., et al., Psychological trauma and functional somatic syndromes: a systematic review and meta-analysis. Psychosom Med, 2014. 76(1): p. 2-11. 
5. Mellon, S.H., et al., Metabolism, Metabolomics, and Inflammation in Posttraumatic Stress Disorder. Biological psychiatry, 2018. 83(10): p. 866-875.

6. Shalev, A., I. Liberzon, and C. Marmar, Post-Traumatic Stress Disorder. N Engl J Med, 2017. 376(25): p. 2459-2469.

7. Zass, L.J., et al., Neuroinflammatory genes associated with post-traumatic stress disorder: implications for comorbidity. Psychiatr Genet, 2017. 27(1): p. 1-16.

8. Sumner, J.A., et al., Posttraumatic Stress Disorder and Inflammation: Untangling Issues of Bidirectionality. Biol Psychiatry, 2020. 87(10): p. 885-897.

9. Pearson-Leary, J., et al., The gut microbiome regulates the increases in depressive-type behaviors and in inflammatory processes in the ventral hippocampus of stress vulnerable rats. Mol Psychiatry, 2020. 25(5): p. 1068-1079.

10. Ha, M. and V.N. Kim, Regulation of microRNA biogenesis. Nat Rev Mol Cell Biol, 2014. 15(8): p. 50924.

11. Bi, Y., G. Liu, and R. Yang, MicroRNAs: novel regulators during the immune response. J Cell Physiol, 2009. 218(3): p. 467-72.

12. Hatfield, S. and H. Ruohola-Baker, microRNA and stem cell function. Cell Tissue Res, 2008. 331(1): p. 57-66.

13. Gauthier, B.R. and C.B. Wollheim, MicroRNAs: 'ribo-regulators' of glucose homeostasis. Nat Med, 2006. 12(1): p. 36-8.

14. Xu, J., et al., miR-124: A Promising Therapeutic Target for Central Nervous System Injuries and Diseases. Cell Mol Neurobiol, 2021.

15. Alsharafi, Walid A., et al., MicroRNA in glutamate receptor-dependent neurological diseases. Clinical Science, 2017. 131(14): p. 1591-1604.

16. Bredy, T.W., et al., MicroRNA regulation of neural plasticity and memory. Neurobiol Learn Mem, 2011. 96(1): p. 89-94.

17. Saraiva, C., M. Esteves, and L. Bernardino, MicroRNA: Basic concepts and implications for regeneration and repair of neurodegenerative diseases. Biochem Pharmacol, 2017. 141: p. 118-131.

18. Manakov, S.A., et al., A Neuronal Transcriptome Response Involving Stress Pathways is Buffered by Neuronal microRNAs. Front Neurosci, 2012. 6: p. 156.

19. Cardoso, A.L., J.R. Guedes, and M.C. de Lima, Role of microRNAs in the regulation of innate immune cells under neuroinflammatory conditions. Curr Opin Pharmacol, 2016. 26: p. 1-9. 
20. Ponomarev, E.D., et al., MicroRNA-124 promotes microglia quiescence and suppresses EAE by deactivating macrophages via the C/EBP-a-PU.1 pathway. Nat Med, 2011. 17(1): p. 64-70.

21. O'Connell, R.M., D.S. Rao, and D. Baltimore, microRNA regulation of inflammatory responses. Annu Rev Immunol, 2012. 30: p. 295-312.

22. Li, Y. and X. Shi, MicroRNAs in the regulation of TLR and RIG-I pathways. Cell Mol Immunol, 2013. 10(1): p. 65-71.

23. Lopez-Ramirez, M.A. and S. Nicoli, Role of miRNAs and epigenetics in neural stem cell fate determination. Epigenetics, 2014. 9(1): p. 90-100.

24. Mishima, T., et al., RT-PCR-based analysis of microRNA (miR-1 and -124) expression in mouse CNS. Brain Res, 2007. 1131(1): p. 37-43.

25. Angelopoulou, E., Y.N. Paudel, and C. Piperi, miR-124 and Parkinson's disease: $A$ biomarker with therapeutic potential. Pharmacol Res, 2019. 150: p. 104515.

26. Soreq, H. and Y. Wolf, NeurimmiRs: microRNAs in the neuroimmune interface. Trends Mol Med, 2011. 17(10): p. 548-55.

27. Smith, P., et al., In vivo regulation of amyloid precursor protein neuronal splicing by microRNAs. J Neurochem, 2011. 116(2): p. 240-7.

28. Han, D., et al., MiR-124 and the Underlying Therapeutic Promise of Neurodegenerative Disorders. Front Pharmacol, 2019. 10: p. 1555.

29. Wan, Y., et al., Postoperative impairment of cognitive function in rats: a possible role for cytokinemediated inflammation in the hippocampus. Anesthesiology, 2007. 106(3): p. 436-43.

30. Chen, Y., et al., miR-124/VAMP3 is a novel therapeutic target for mitigation of surgical traumainduced microglial activation. Signal Transduct Target Ther, 2019. 4: p. 27.

31. Chivero, E.T., et al., Engineered Extracellular Vesicles Loaded With miR-124 Attenuate CocaineMediated Activation of Microglia. Front Cell Dev Biol, 2020. 8: p. 573.

32. Nie, P.Y., et al., miR-142 downregulation alleviates rat PTSD-like behaviors, reduces the level of inflammatory cytokine expression and apoptosis in hippocampus, and upregulates the expression of fragile $X$ mental retardation protein. J Neuroinflammation, 2021. 18(1): p. 17.

33. Walsh, M.C., J. Lee, and Y. Choi, Tumor necrosis factor receptor- associated factor 6 (TRAF6) regulation of development, function, and homeostasis of the immune system. Immunol Rev, 2015. 266(1): p. 72-92. 
34. Dou, Y., et al., Roles of TRAF6 in Central Nervous System. Curr Neuropharmacol, 2018. 16(9): p. 1306-1313.

35. Jakus, P.B., et al., TRAF6 is functional in inhibition of TLR4-mediated NF-KB activation by resveratrol. J Nutr Biochem, 2013. 24(5): p. 819-23.

36. Chandel, N.S., P.T. Schumacker, and R.H. Arch, Reactive oxygen species are downstream products of TRAF-mediated signal transduction. J Biol Chem, 2001. 276(46): p. 42728-36.

37. Li, T., et al., The Ubiquitin E3 Ligase TRAF6 Exacerbates Ischemic Stroke by Ubiquitinating and Activating Rac1. J Neurosci, 2017. 37(50): p. 12123-12140.

38. Nataf, S., M. Guillen, and L. Pays, Common Neurodegeneration-Associated Proteins Are Physiologically Expressed by Human B Lymphocytes and Are Interconnected via the Inflammation/Autophagy-Related Proteins TRAF6 and SQSTM1. Front Immunol, 2019. 10: p. 2704.

39. Feng, $\mathrm{H}$. , et al., EGFR phosphorylation of DCBLD2 recruits TRAF6 and stimulates AKT-promoted tumorigenesis. J Clin Invest, 2014. 124(9): p. 3741-56.

40. Liberzon, I., M. Krstov, and E.A. Young, Stress-restress: effects on ACTH and fast feedback. Psychoneuroendocrinology, 1997. 22(6): p. 443-53.

41. Shi, J.H. and S.C. Sun, Tumor Necrosis Factor Receptor-Associated Factor Regulation of Nuclear Factor KB and Mitogen-Activated Protein Kinase Pathways. Front Immunol, 2018. 9: p. 1849.

42. Kapoor, M., et al., Effect of the NADPH oxidase inhibitor apocynin on ischemia-reperfusion hippocampus injury in rat brain. Biomed Pharmacother, 2018. 97: p. 458-472.

43. Vincent, S.R. and B.T. Hope, Neurons that say NO. Trends Neurosci, 1992. 15(3): p. 108-13.

44. Contestabile, A. and E. Ciani, Role of nitric oxide in the regulation of neuronal proliferation, survival and differentiation. Neurochem Int, 2004. 45(6): p. 903-14.

45. Cossenza, M., et al., Nitric oxide in the nervous system: biochemical, developmental, and neurobiological aspects. Vitam Horm, 2014. 96: p. 79-125.

46. Ehrlich, l., et al., PSD-95 is required for activity-driven synapse stabilization. Proc Natl Acad Sci U S A, 2007. 104(10): p. 4176-81.

47. Cesca, F., et al., The synapsins: key actors of synapse function and plasticity. Prog Neurobiol, 2010. 91(4): p. 313-48.

48. Lynch, M.A., Long-term potentiation and memory. Physiol Rev, 2004. 84(1): p. 87-136. 
49. Zhu, Y., et al., Interleukin-10 Protection against Lipopolysaccharide-Induced Neuro-Inflammation and Neurotoxicity in Ventral Mesencephalic Cultures. Int J Mol Sci, 2015. 17(1).

50. Dutta, G., P. Zhang, and B. Liu, The lipopolysaccharide Parkinson's disease animal model: mechanistic studies and drug discovery. Fundam Clin Pharmacol, 2008. 22(5): p. 453-64.

51. Sharon, G., et al., The Central Nervous System and the Gut Microbiome. Cell, 2016. 167(4): p. 915932.

52. Thion, M.S., et al., Microbiome Influences Prenatal and Adult Microglia in a Sex-Specific Manner. Cell, 2018. 172(3): p. 500-516.e16.

53. Benakis, C., et al., The microbiome-gut-brain axis in acute and chronic brain diseases. Curr Opin Neurobiol, 2020. 61: p. 1-9.

54. Veremeyko, T., et al., IL-4/IL-13-dependent and independent expression of miR-124 and its contribution to M2 phenotype of monocytic cells in normal conditions and during allergic inflammation. PLoS One, 2013. 8(12): p. e81774.

55. Song, Y., et al., M2 microglia-derived exosomes protect the mouse brain from ischemia-reperfusion injury via exosomal miR-124. Theranostics, 2019. 9(10): p. 2910-2923.

56. Fischbach, S.J. and T.J. Carew, MicroRNAs in memory processing. Neuron, 2009. 63(6): p. 714-6.

57. Aalaei-Andabili, S.H. and N. Rezaei, MicroRNAs (MiRs) Precisely Regulate Immune System Development and Function in Immunosenescence Process. Int Rev Immunol, 2016. 35(1): p. 57-66.

58. Wang, X., et al., A Novel MicroRNA-124/PTPN1 Signal Pathway Mediates Synaptic and Memory Deficits in Alzheimer's Disease. Biol Psychiatry, 2018. 83(5): p. 395-405.

59. Zhou, Y., et al., Role of Post-Transcriptional Control of Calpain by miR-124-3p in the Development of Alzheimer's Disease. J Alzheimers Dis, 2019. 67(2): p. 571-581.

60. Dong, R.F., et al., The Neuroprotective Role of MiR-124-3p in a 6-Hydroxydopamine-Induced Cell Model of Parkinson's Disease via the Regulation of ANAX5. J Cell Biochem, 2018. 119(1): p. 269-277.

61. Yao, L., et al., MicroRNA-124 regulates the expression of MEKK3 in the inflammatory pathogenesis of Parkinson's disease. J Neuroinflammation, 2018. 15(1): p. 13.

62. Lee, S.T., et al., Exosome-Based Delivery of miR-124 in a Huntington's Disease Model. J Mov Disord, 2017. 10(1): p. 45-52.

63. Johnson, R. and N.J. Buckley, Gene dysregulation in Huntington's disease: REST, microRNAs and beyond. Neuromolecular Med, 2009. 11(3): p. 183-99. 
64. Deo, M., et al., Detection of mammalian microRNA expression by in situ hybridization with RNA oligonucleotides. Dev Dyn, 2006. 235(9): p. 2538-48.

65. Krichevsky, A.M., et al., Specific microRNAs modulate embryonic stem cell-derived neurogenesis. Stem Cells, 2006. 24(4): p. 857-64.

66. Periyasamy, P., et al., Cocaine-Mediated Downregulation of miR-124 Activates Microglia by Targeting KLF4 and TLR4 Signaling. Mol Neurobiol, 2018. 55(4): p. 3196-3210.

67. Yang, Y., et al., Luteolin alleviates neuroinflammation via downregulating the TLR4/TRAF6/NF-KB pathway after intracerebral hemorrhage. Biomed Pharmacother, 2020. 126: p. 110044.

68. Ma, Q., et al., Proteasome-independent polyubiquitin linkage regulates synapse scaffolding, efficacy, and plasticity. Proc Natl Acad Sci U S A, 2017. 114(41): p. E8760-E8769.

69. Vemula, S.K., et al., The Interaction of TRAF6 With Neuroplastin Promotes Spinogenesis During Early Neuronal Development. Front Cell Dev Biol, 2020. 8: p. 579513.

70. Dilshara, M.G., et al., Downregulation of NO and PGE2 in LPS-stimulated BV2 microglial cells by trans-isoferulic acid via suppression of PI3K/Akt-dependent NF-KB and activation of Nrf2-mediated HO-1. Int Immunopharmacol, 2014. 18(1): p. 203-11.

71. Park, H.Y., et al., Purpurogallin exerts anti-inflammatory effects in lipopolysaccharide-stimulated $B V 2$ microglial cells through the inactivation of the NF-KB and MAPK signaling pathways. Int J Mol Med, 2013. 32(5): p. 1171-8.

72. Song, F., et al., Schizandrin A Inhibits Microglia-Mediated Neuroninflammation through Inhibiting TRAF6-NF-KB and Jak2-Stat3 Signaling Pathways. PLoS One, 2016. 11(2): p. e0149991.

73. Stellwagen, D. and R.C. Malenka, Synaptic scaling mediated by glial TNF-alpha. Nature, 2006. 440(7087): p. 1054-9.

74. Bezzi, P., et al., CXCR4-activated astrocyte glutamate release via TNFalpha: amplification by microglia triggers neurotoxicity. Nat Neurosci, 2001. 4(7): p. 702-10.

75. González-Burgos, I., From synaptic transmission to cognition: an intermediary role for dendritic spines. Brain Cogn, 2012. 80(1): p. 177-83.

76. González-Ramírez, M.M., et al., Changes in the plastic properties of hippocampal dendritic spines underlie the attenuation of place learning in healthy aged rats. Neurobiol Learn Mem, 2014. 109: p. 94103.

77. Cingolani, L.A. and Y. Goda, Actin in action: the interplay between the actin cytoskeleton and synaptic efficacy. Nat Rev Neurosci, 2008. 9(5): p. 344-56. 


\section{Figures}
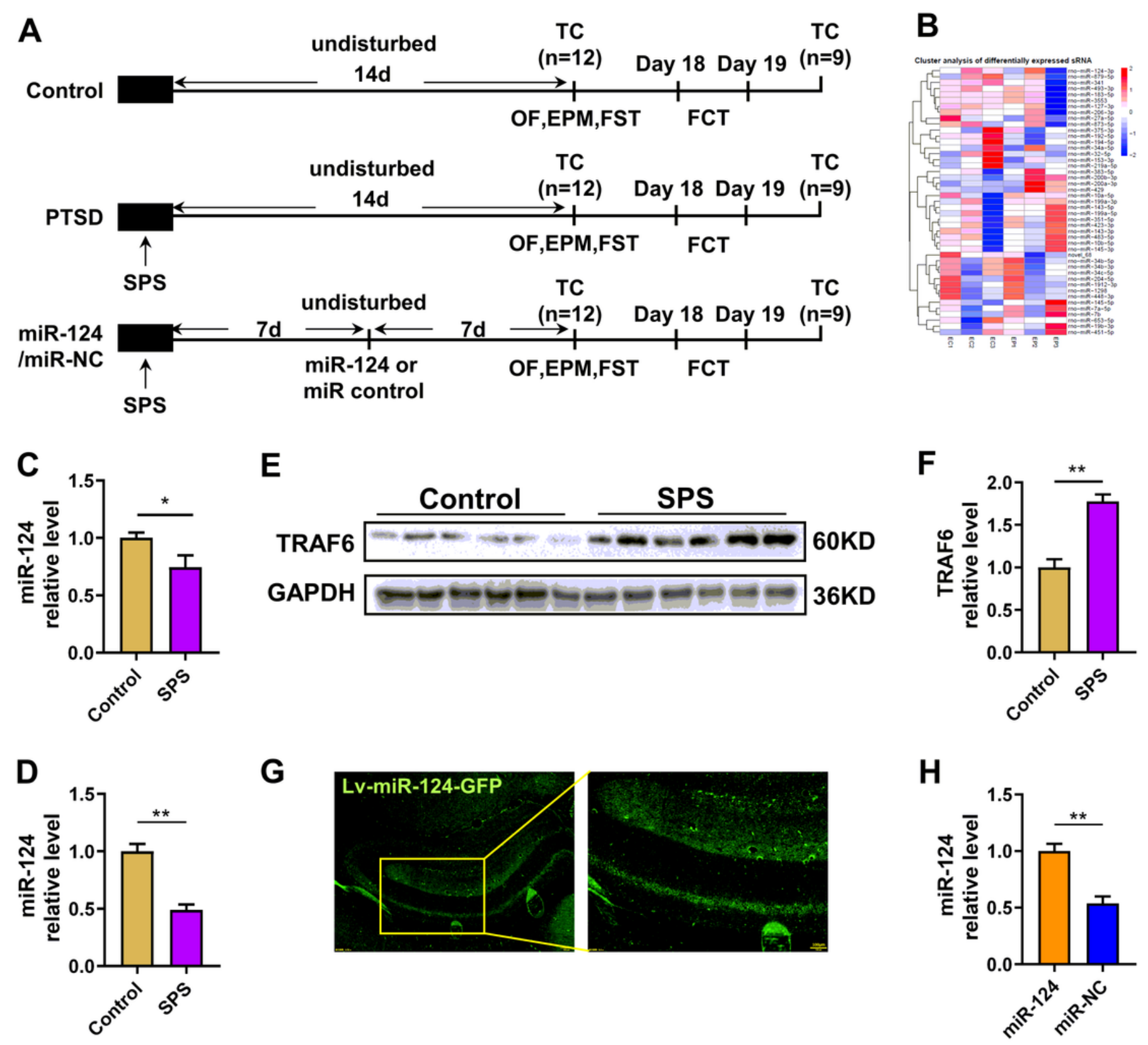

Figure 1

The levels of miR-124 and TRAF6 in the hippocampus of rats exposed to SPS. a Schematic of the procedure and timeline in PTSD experiments. $\mathbf{b}$ The clustering map showed that the level of microRNAs and $\mathbf{c}$ the quantitation of expression of miR-124-3p in hippocampus of rats in control or SPS group by high-throughput sequencing analysis. $\mathbf{d}$ The relative level of miR-124-3p in the hippocampus of rats in control or SPS group tested by RT-qPCR. e The representative bands of TRAF6 and GAPDH measured by western blot. GAPDH was used as a loading control. $\mathbf{f}$ The relative expression of TRAF6 in the hippocampus of rats in control or SPS group. $\mathbf{g}$ The expression of the GFP reporter in the hippocampus 
one week after administration of miR-124-3p delivered by the lentivirus (LV-miR-124). $\mathbf{h}$ Relative level of miR-124 in the hippocampus one week after administration of LV-miR-124 compared with that of LVcontrol. $n=3$ per genotype in high-throughput sequencing analysis and $n=6$ rats per genotype in RTqPCR or western blot. Bar graphs represent the mean \pm SEM of independent experimental triplicates. Oneway ANOVA followed by Bonferroni's post hoc test; $* P<0.05,{ }^{\star *} P<0.01$

A

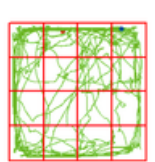

Control

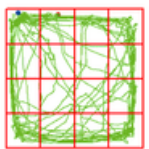

miR-124

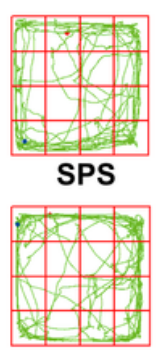

miR-NC
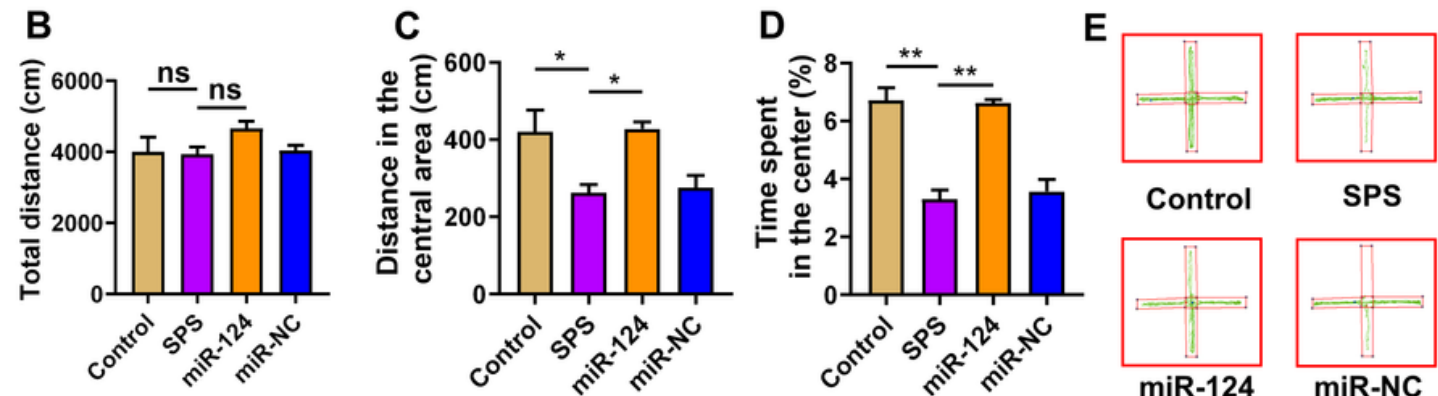

SPS

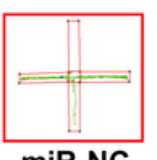

miR-NC

$\mathbf{F}$
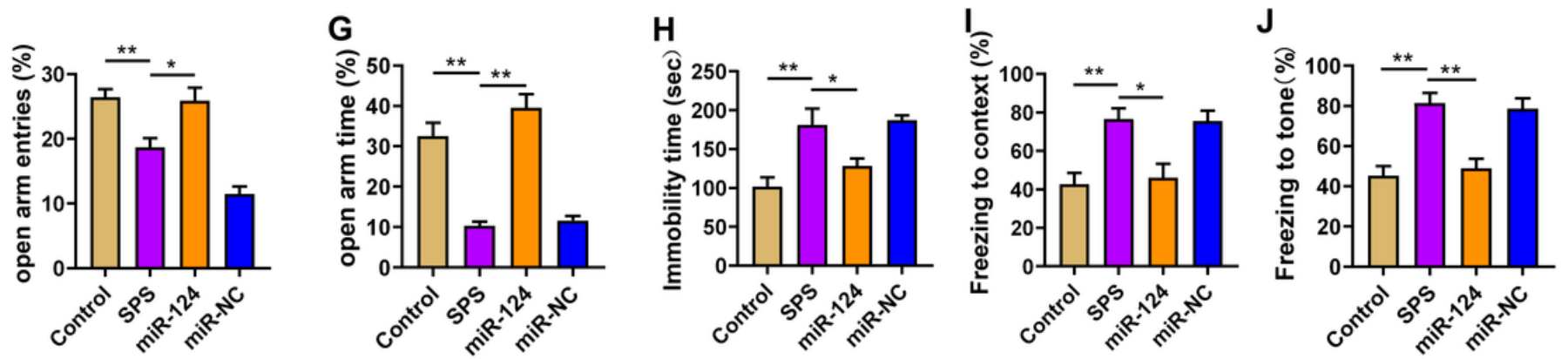

\section{Figure 2}

MiR-124 alleviated the PTSD-like behavior in rats exposed to the SPS procedure. a Representative travel pathway of rats in each group in OFT. $\mathbf{b}$ Total distance, $\mathbf{c}$ distance in the central area, and $\mathbf{d}$ the percentage of time spent in the center of rats in each group in the OFT. e Representative moving trajectory of rats in each group in the EPM test. $\mathbf{f}$ Comparison of time spent in open arm time and $\mathbf{g}$ open arm entries of rats in each group in the EPM test. $\mathbf{h}$ The immobility time of rats in each group in FST. $\mathbf{i}$ Quantitation of the freezing time to context and $\mathbf{j}$ tone of rats in each group. $\mathrm{n}=6$ rats per genotype. Bar graphs represent the mean \pm SEM of independent experimental triplicates. One-way ANOVA followed by Bonferroni's post hoc test; ${ }^{*} P<0.05,{ }^{*} P<0.01$ 

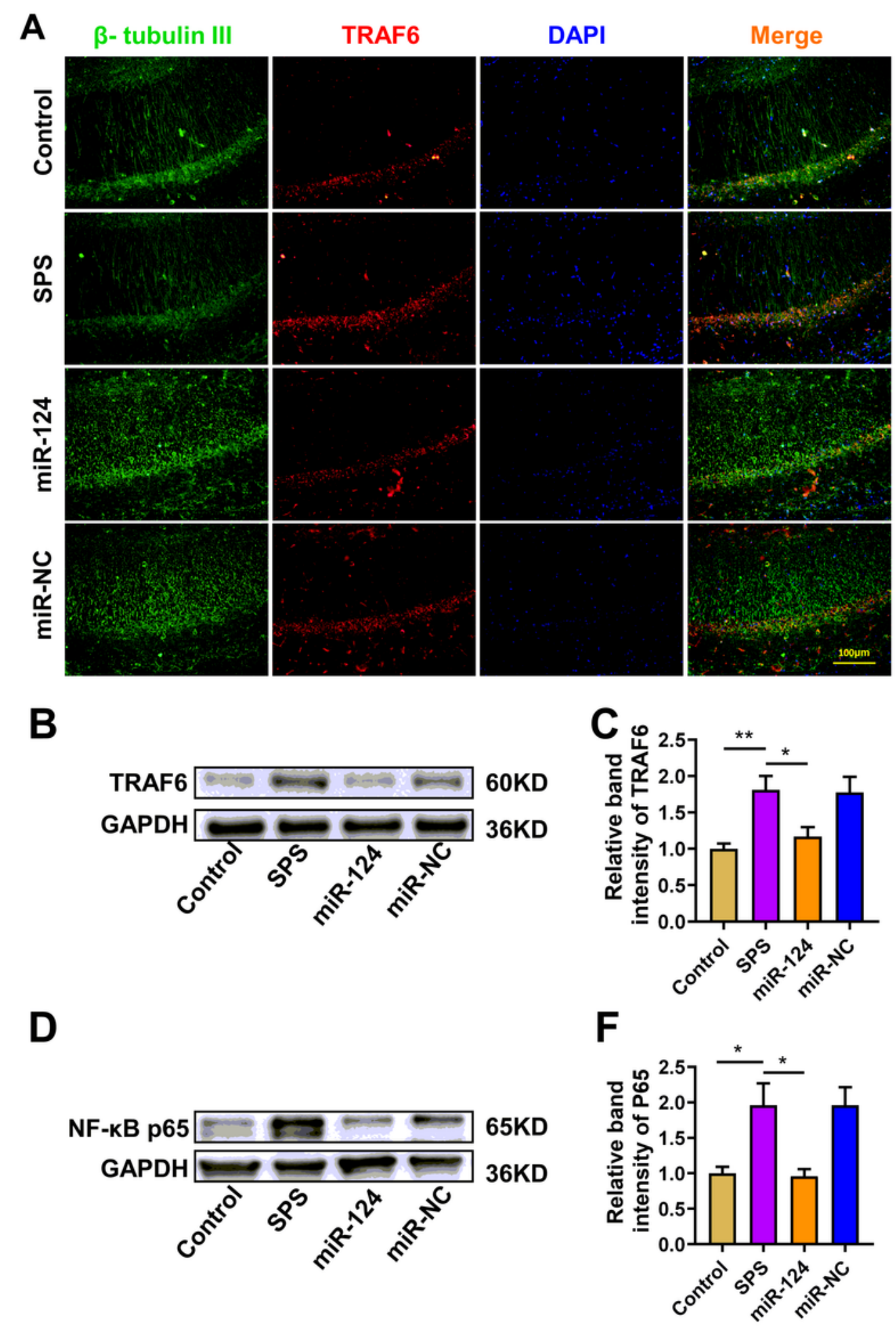

Figure 3

MiR-124 decreased the level of TRAF6 in the hippocampus of SPS rats. a Representative image indicating TRAF6 (red) and $\beta$-tubulin $Ш$ (green) positive cells in rat hippocampus of each group. $\mathbf{b}$ The relative expression of TRAF6 measured by western blot analysis in rat hippocampus of each group. c The quantitation of relative band intensity of TRAF6 protein in each group. $d$ The representative images of NFKB p65 and GAPDH tested by western blot. e The quantitation of relative band intensity of NF-kB p65 
protein in each group. Sample western blots for each protein are presented with GAPDH as a loading control. $\mathrm{n}=6$ rats per genotype. Bar graphs represent the mean \pm SEM of independent experimental triplicates. One-way ANOVA followed by Bonferroni's post hoc test; ${ }^{*} P<0.05,{ }^{*} P<0.01$
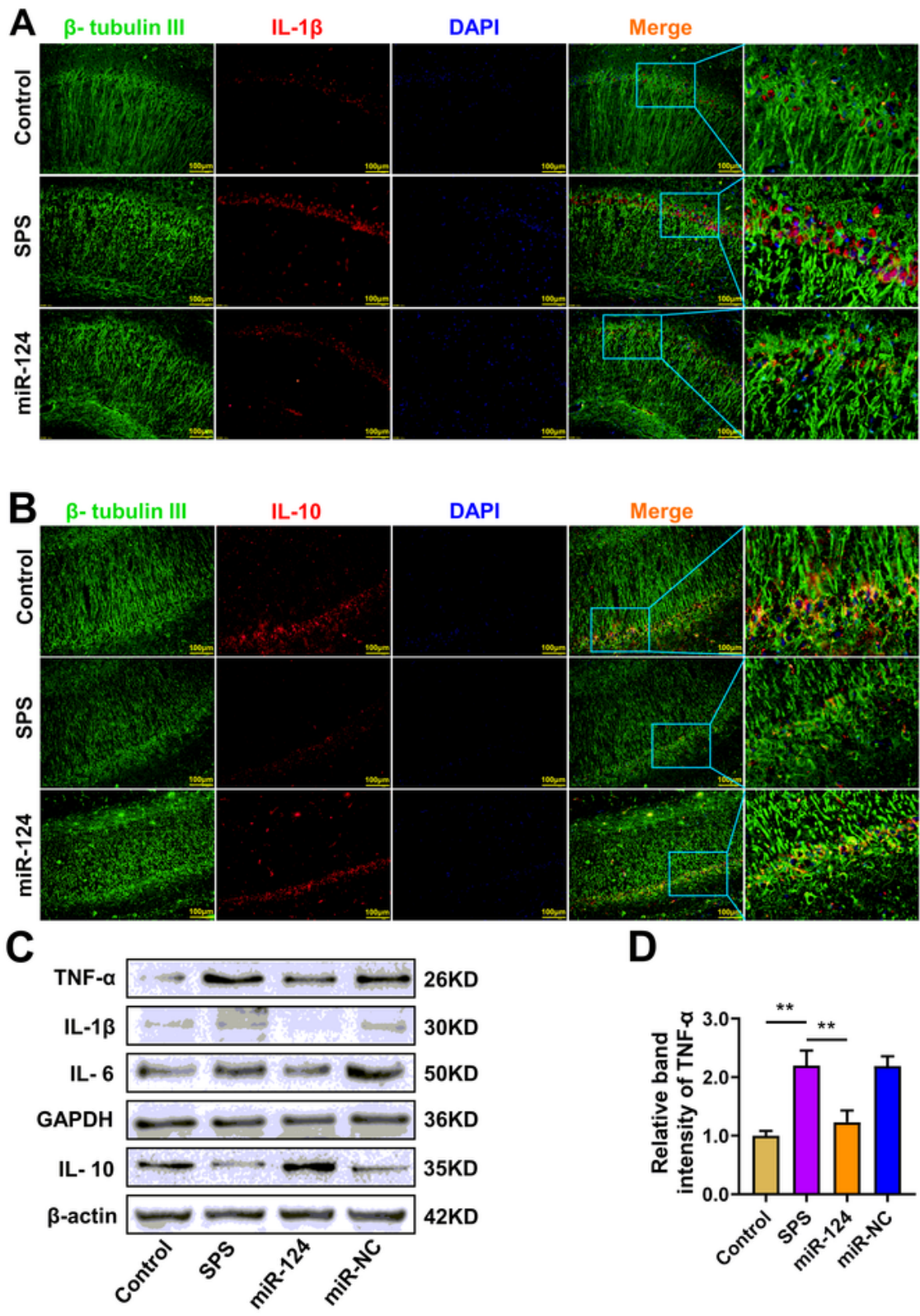

E

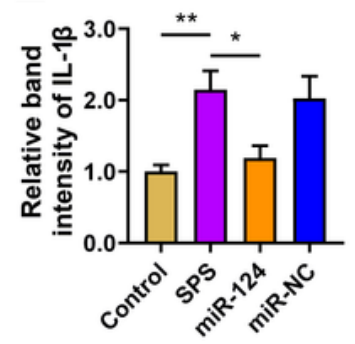

DAPI

Merge

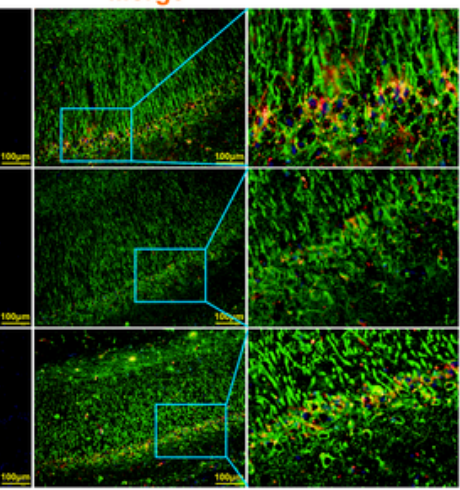

D

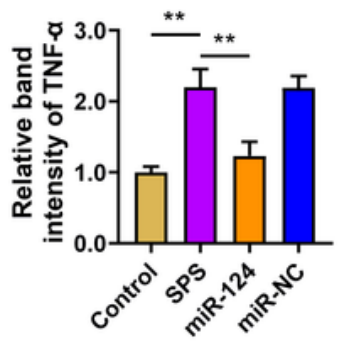

G

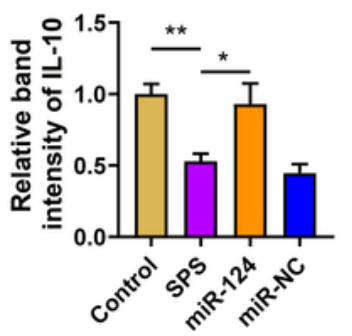

Figure 4 
MiR-124 regulated the level of inflammatory cytokines in the hippocampus of rats exposed to SPS procedure. Representative image indicating a IL-1 $\beta$ (red) and $\beta$-tubulin $Ш$ (green), and b IL-10 (red) and $\beta$ tubulin $Ш$ (green) positive cells in rat hippocampus of each group. $\mathbf{c}$ The representative bands of TNF- $\alpha$, IL-1 $1 \beta, \mathrm{IL}-6$ and IL-10 measured by western blot analysis in rat hippocampus of each group. The quantitation of relative band intensity of $\mathbf{d}$ TNF-a, e IL-1 $\beta$, $\mathbf{f}$ IL- 6 , and $\mathbf{g}$ IL-10 protein in each group. Sample western blots for TNF- $a$, IL-1 $\beta$ and IL- 6 were presented with GAPDH, while IL-10 was presented with $\beta$ actin as a loading control. $\mathrm{n}=6$ rats per genotype. Bar graphs represent the mean \pm SEM of independent experimental triplicates. One-way ANOVA followed by Bonferroni's post hoc test; ${ }^{*} P<0.05, * \star P<0.01$

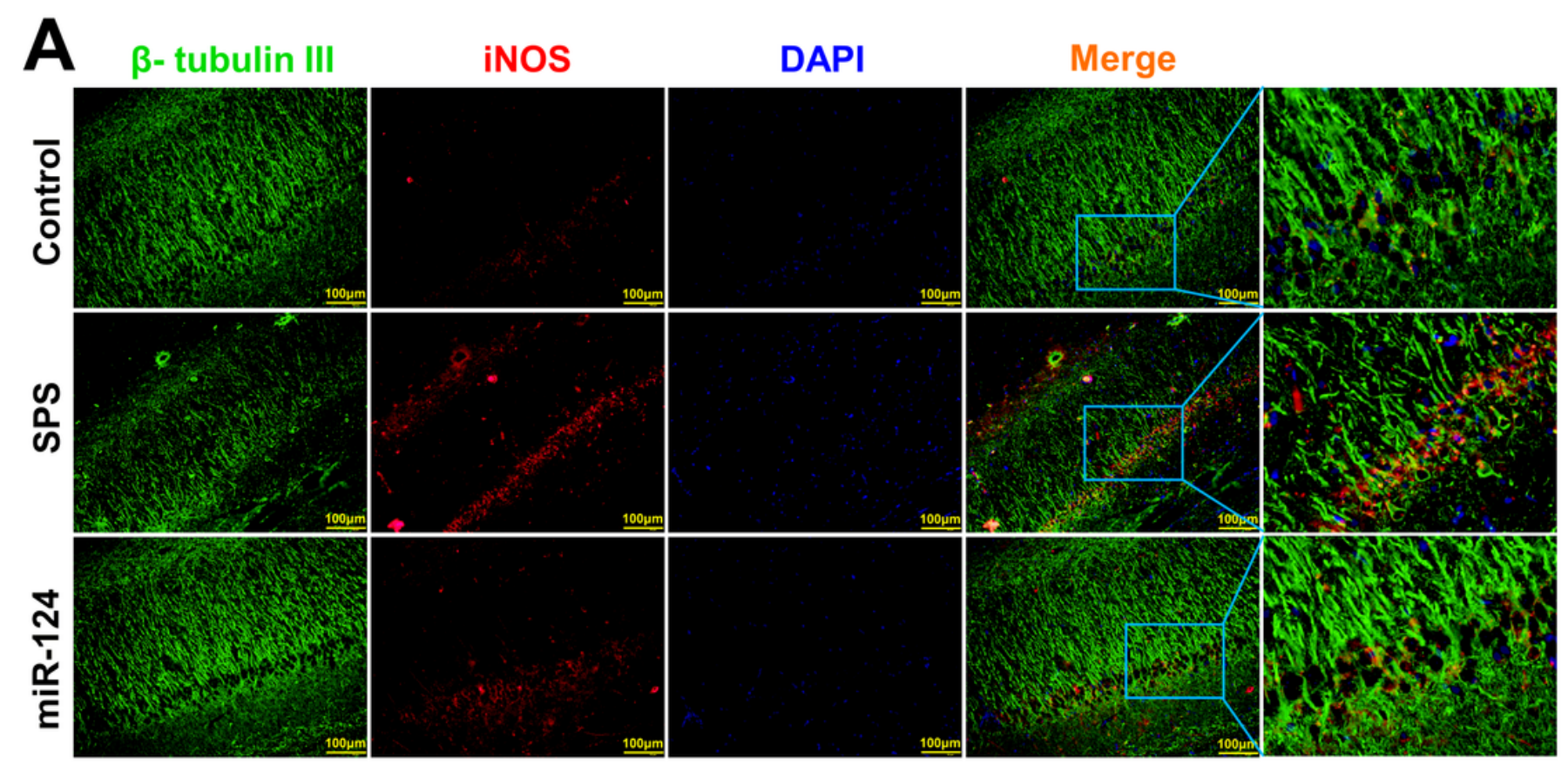

B

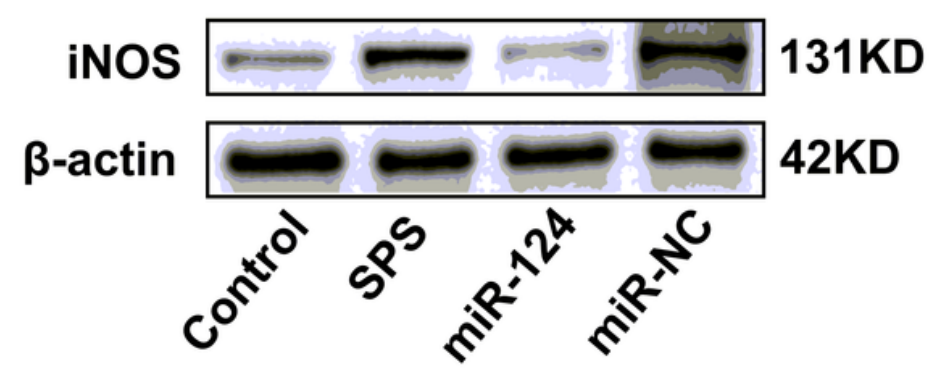

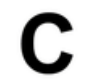

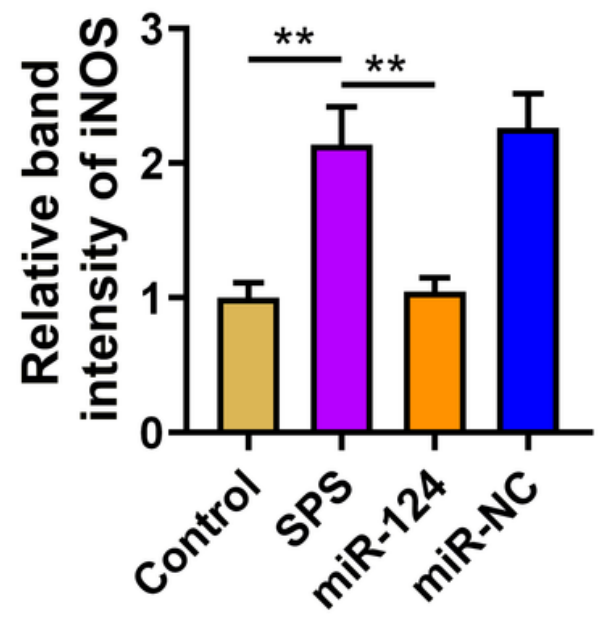

Figure 5 
MiR-124 decreased the level of iNOS in the hippocampus of SPS rats. a Representative image indicating iNOS (red) and $\beta$-tubulin Ш(green) positive cells in rat hippocampus of each group. $b$ The relative images of iNOS and GAPDH tested by western blot analysis in rat hippocampus of each group. $c$ The quantitation of relative band intensity of iNOS protein in each group. Sample western blots for each protein are presented with GAPDH as a loading control. $n=6$ rats per genotype. Bar graphs represent the mean \pm SEM of independent experimental triplicates. One-way ANOVA followed by Bonferroni's post hoc test; $* \star P<0.01$
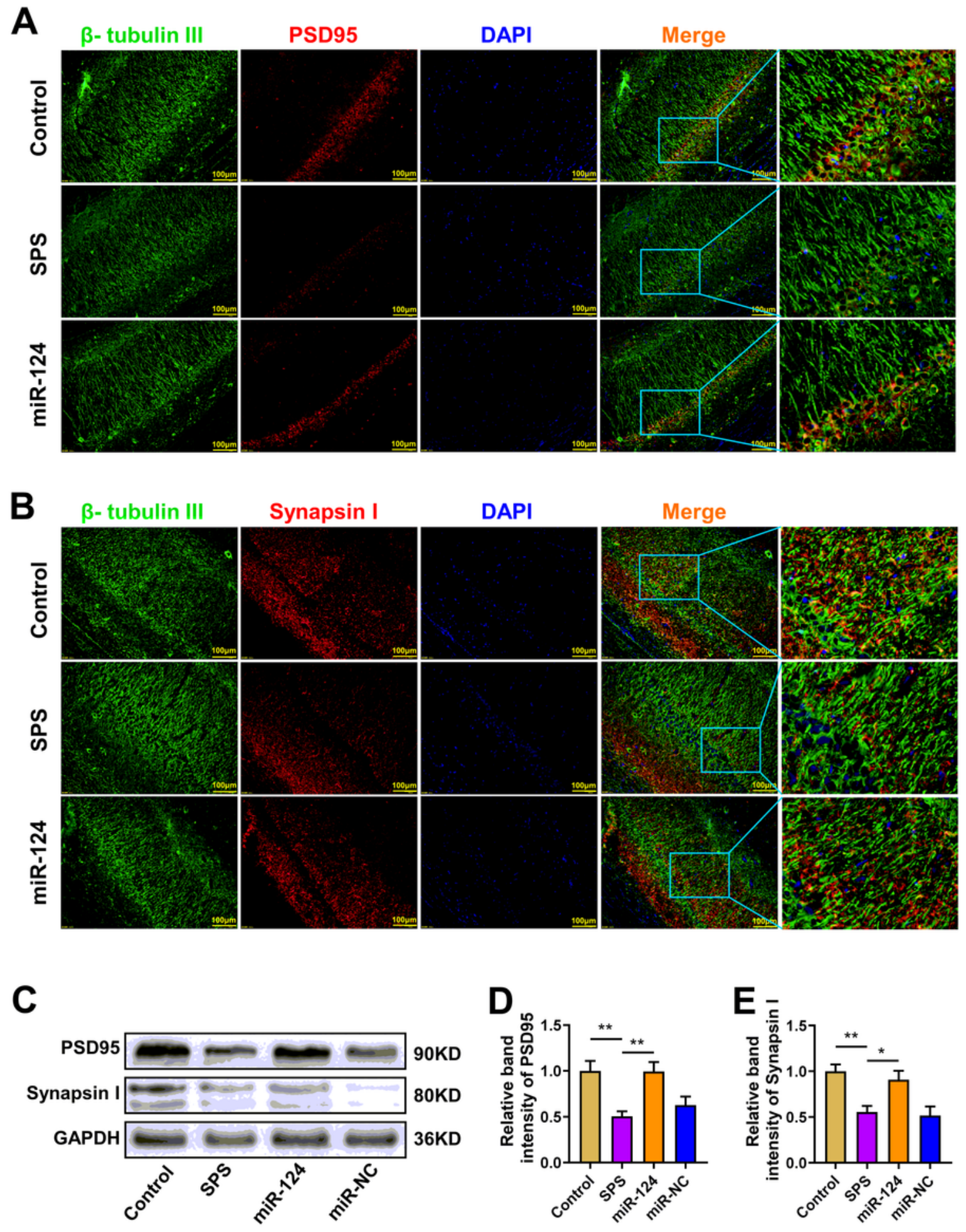
Figure 6

MiR-124 upregulated the expression level of PSD95 and synapsin I in the hippocampus of rats exposed to SPS procedure. Representative image indicating a PSD95 (red) and $\beta$-tubulin $Ш$ (green), and $\mathbf{b}$ Synapsin I (red) and $\beta$-tubulin $Ш$ (green) positive cells in four groups. $\mathbf{c}$ The relative expression of PSD95 and Synapsin I measured by western blot analysis in each group. The relative band intensity of $\mathbf{d}$ PSD 95 and e Synapsin I protein in each group. Sample western blots for each protein are presented with GAPDH as a loading control. $n=6$ rats per genotype. Bar graphs represent the mean \pm SEM of independent experimental triplicates. One-way ANOVA followed by Bonferroni's post hoc test; ${ }^{\star} P<0.05,{ }^{\star \star} P<0.01$

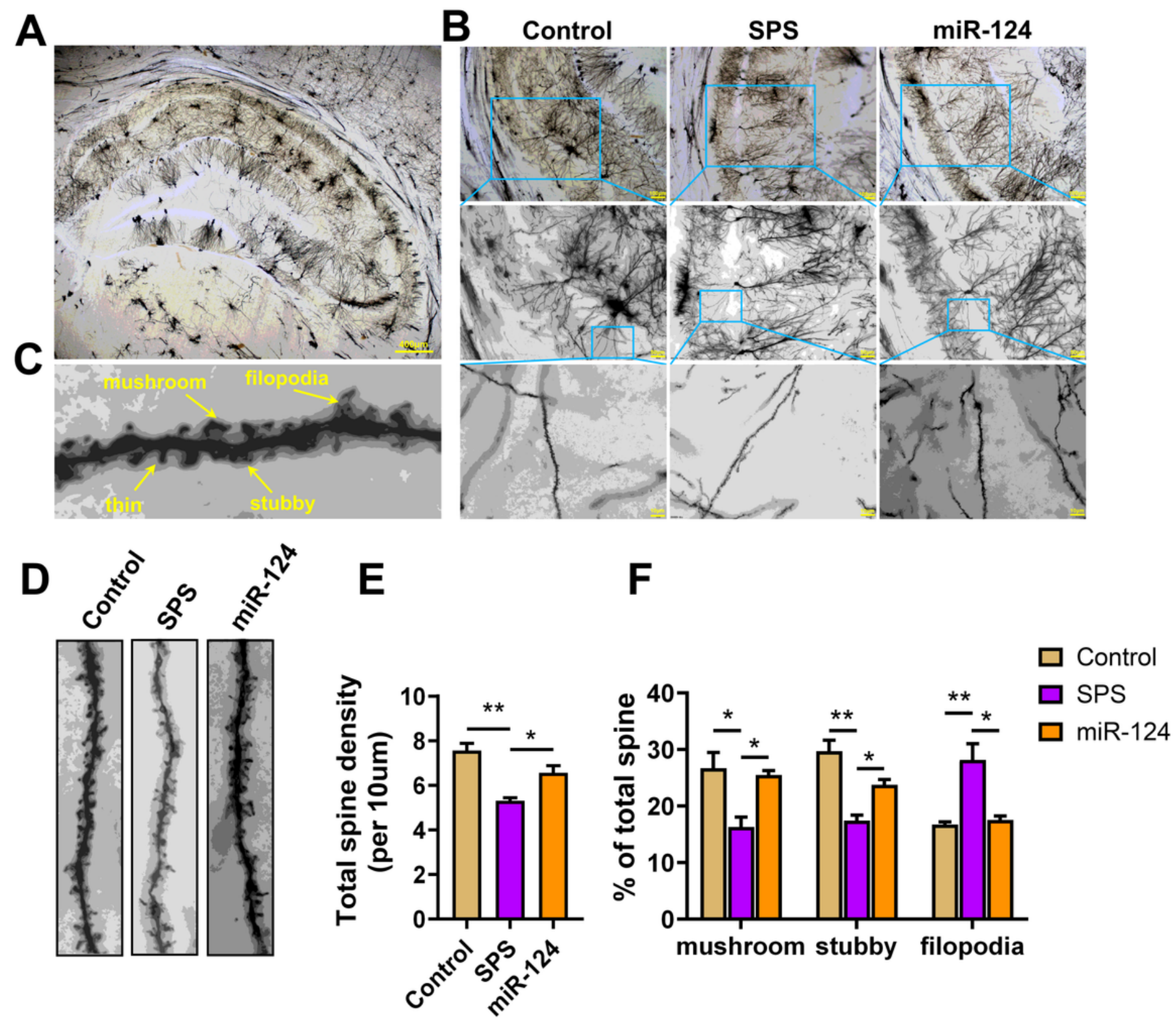

Figure 7 


\section{The effects of miR-124 treatment on spine density in hippocampus in rat exposed to SPS procedure. a}

Representative microphotograph showed Golgi-Cox staining pyramidal neurons in the hippocampus. Scale bar $=400 \mu \mathrm{m}$ b Representative images of neurons in the hippocampus from control, SPS or miR124. c Sample spines from segment in control group and were divided into filopodia, thin, stubby and mushroom types according to their size and shape. $\mathbf{d}$ Representative images of Golgi-Cox staining basilar dendrites. Number of spines was counted from basilar dendrites per $10 \mu \mathrm{m}$. The quantification of e spine density and $\mathbf{f}$ number of spine type in each group. $n=3$ rats per genotype. Bar graphs represent the mean \pm SEM of independent experimental triplicates. One-way ANOVA followed by Bonferroni's post hoc test; ${ }^{*} P$ $<0.05, * \star P<0.01$ 


\section{Control}

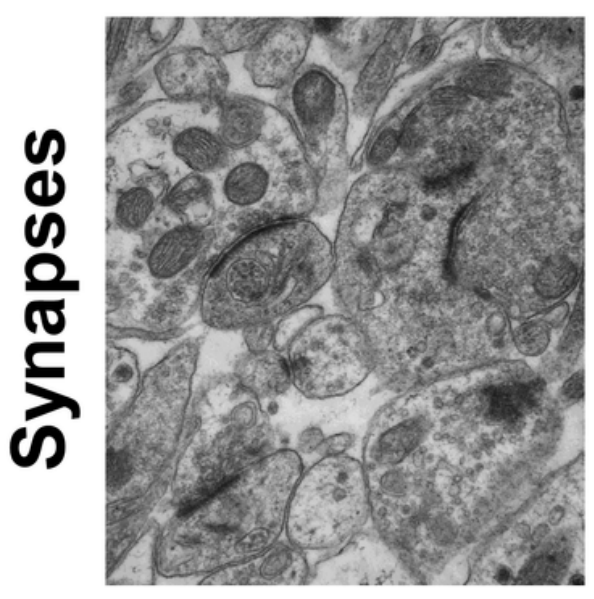

\section{SPS}

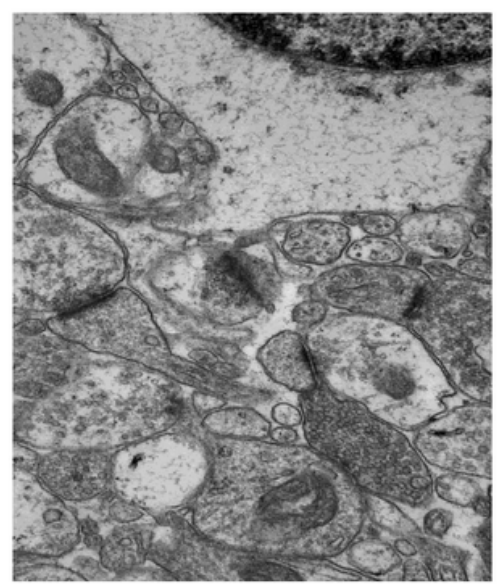

miR-124

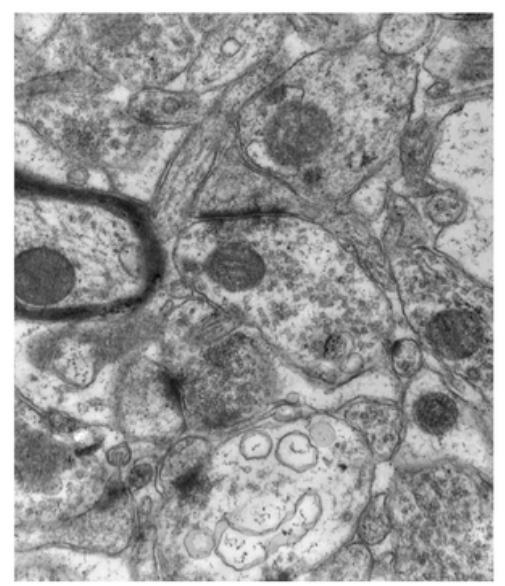

\section{B}

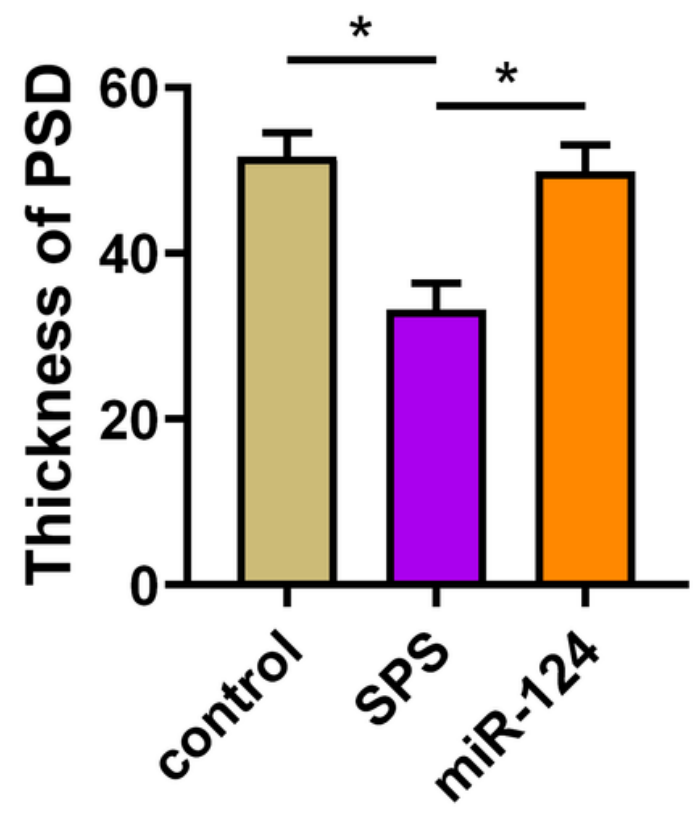

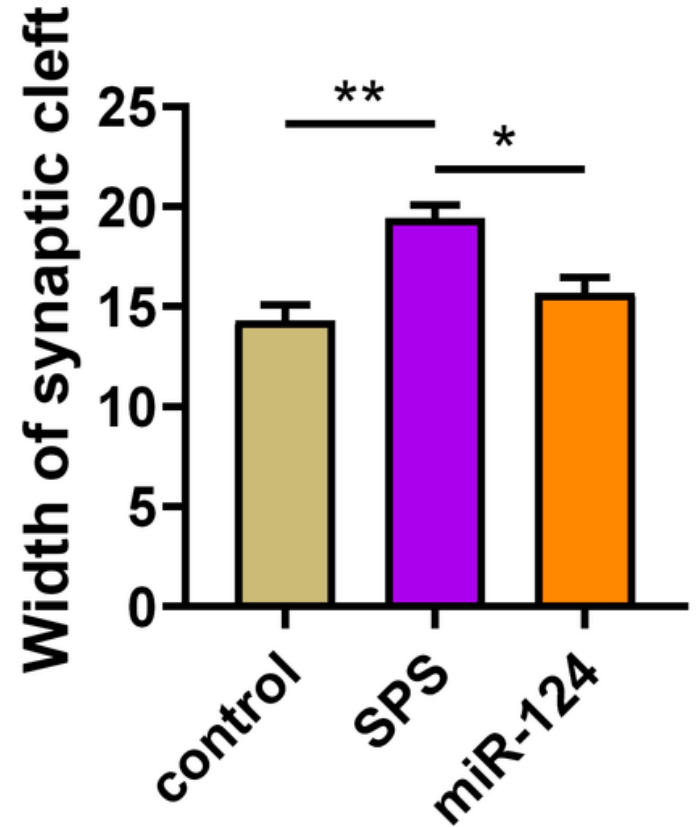

Figure 8

The effects of miR-124 treatment on the synaptic ultrastructure in hippocampus in rat exposed to SPS procedure. a Representative image of synapse structure in control, SPS or miR-124 group. The quantification of $\mathbf{b}$ thickness of PSD and $\mathbf{c}$ width of synaptic cleft in each group. $n=3$ rats per genotype. Bar graphs represent the mean \pm SEM of independent experimental triplicates. One-way ANOVA followed by Bonferroni's post hoc test; ${ }^{*} P<0.05,{ }^{\star *} P<0.01$ 


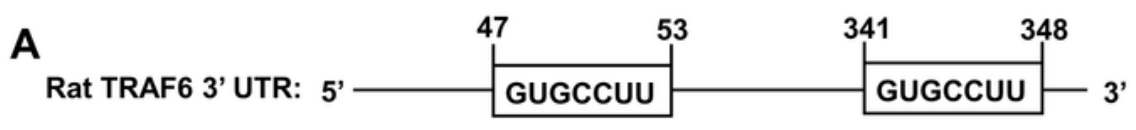

Rno-miR-124-3p: 3' ----- CCGUAAGUGGCGCACGGAAU ----- 5'

Mutated 3' UTR : $\quad$ 5' ----- AAAACCAAGGCACCC ----- TACTGTAAGGCACAA ----- 3'

B

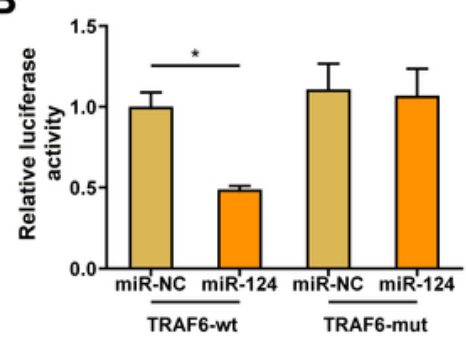

$\mathbf{E}$
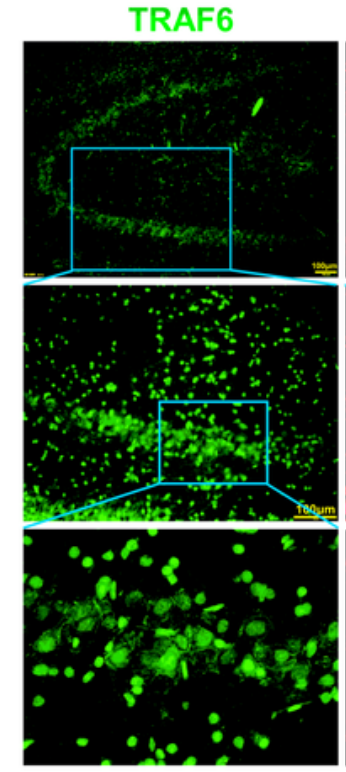

C

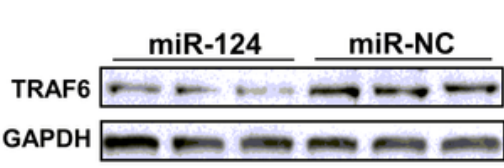

miR-124
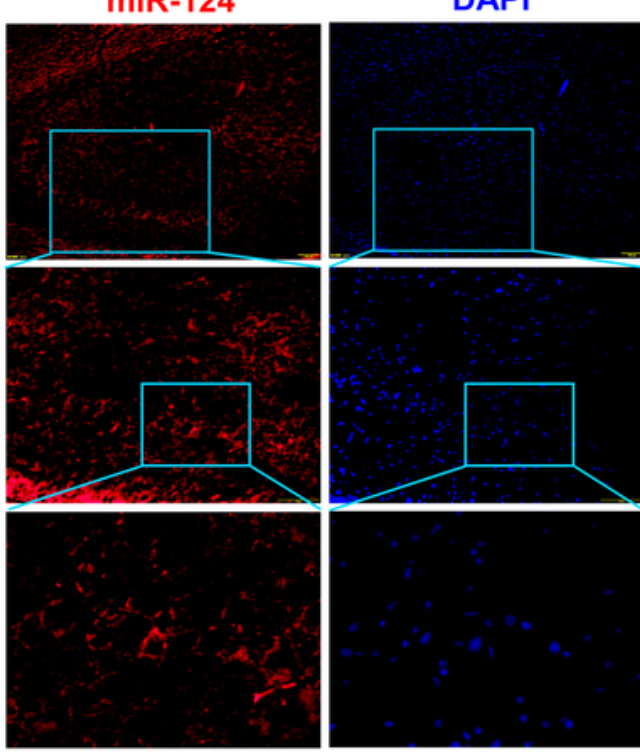

D

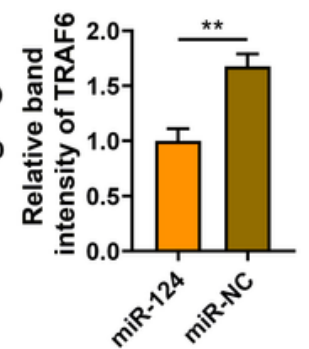

Merge

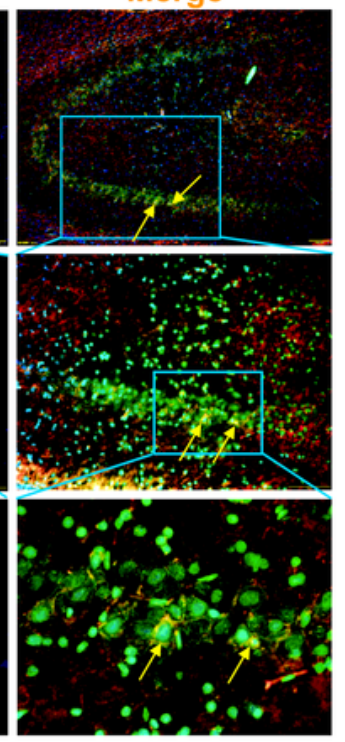

$\mathbf{F}$

G

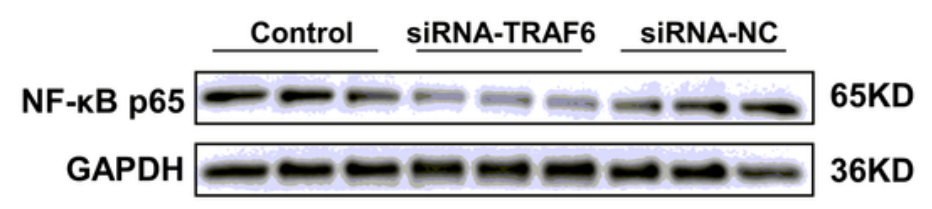

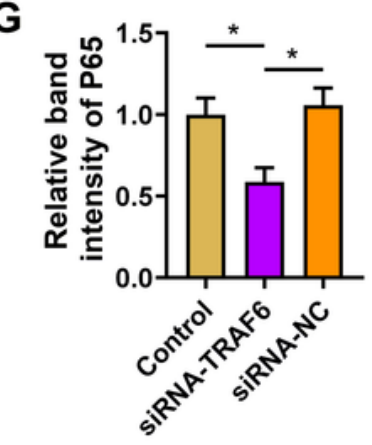

Figure 9

miR-124 directly targets TRAF6 3'UTR and regulates NF-kB. a Schematic illustration indicating the conserved seed match of TRAF6 3'UTR to miR-124-3p. The putative binding sites were predicted by TargetScan. b Relative luciferase activity expressed with firefly/Renilla luciferase activity. Luciferase was fused to the 3'UTR of TRAF6 containing wild-type or a mutated seed sites in the presence of miR-control or miR-124-3p. c Representative images and $\mathbf{d}$ the quantitation of relative band intensity of TRAF6 
measured by western blot in neuron-like cells of the miR-124 or miR-NC groups. e Rrna-FISH of location between miR-124-3p and TRAF6 in hippocampus tissues. $f$ Representative images and $\mathbf{g}$ the quantitation of relative band intensity of NF-KB p65 tested by western blot in neuro-like cells of each group. Sample western blots for each protein are presented with GAPDH as a loading control. Bar graphs represent the mean \pm SEM of independent experimental triplicates. One-way ANOVA followed by Bonferroni's post hoc test; $* P<0.05, * \star P<0.01$ 